\title{
On the resolvents of dyadic paraproducts
}

María Cristina Pereyra

\section{Introduction.}

We consider the boundedness of certain singular integral operators that arose in the study of Sobolev spaces on Lipschitz curves, [P1]. The standard theory available (David and Journés $T 1$ Theorem, for instance; see [D]) does not apply to this case because the operators

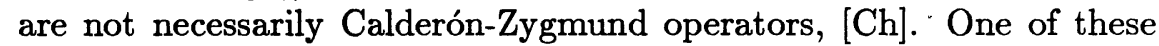
operators gives an explicit formula for the resolvent at $\lambda=1$ of the dyadic paraproduct, [Ch].

The operators in question can be thought of as multipliers for a wavelet basis, $[\mathrm{M}]$. In particular, given a function in $L^{p}\left(\mathbb{R}^{n}\right)$, we can write its decomposition in the Haar basis. If we want to perturb the coefficients, we know that we can multiply by a bounded sequence of numbers and preserve the norm. Suppose instead that we multiply each Haar coefficient by a function. What are the necessary and sufficient conditions for such an operator to be bounded in $L^{p}\left(\mathbb{R}^{n}\right)$ ?

Our model operator will be

$$
T f(x)=\sum_{j} \omega_{j}(x) \Delta_{j} f(x),
$$

where $\Delta_{j} f$ denotes the Haar decomposition of $f$ at level $j$, and the $\omega_{j}$ 's are functions.

Given a doubling dyadic weight $\omega$ (i.e. a positive locally integrable function such that $\omega(\tilde{Q}) \leq C \omega(Q)$, for every dyadic cube $Q, \tilde{Q}$ its 
parent, and where $\left.\omega(Q)=\int_{Q} \omega\right)$. Consider the non trivial examples

$$
\omega_{j}(x)=\frac{\omega(x)}{E_{j} \omega(x)} \quad \text { or } \quad \omega_{j}(x)=\frac{\omega(x)}{E_{j+1} \omega(x)},
$$

where we define $E_{j} \omega(x)=\omega(Q) /|Q|, x \in Q \in \mathcal{D}_{j}$, and $\mathcal{D}_{j}$ denotes the dyadic cubes of side length $2^{-j},|Q|$ denotes the Lebesgue measure of $Q$. Denote by $T_{\omega}$ and $P_{\omega}$ the operators corresponding to the first and second sequence of weights respectively. In Section 3 we give necessary and sufficient conditions for the boundedness of such operators, more precisely, we will prove

Theorem I. Given a dyadic doubling weight $\omega, 1<p<\infty, 1 / p+1 / q=$ 1 , the following properties are equivalent,

i) $T_{\omega}$ is bounded in $L^{p}\left(\mathbb{R}^{n}\right)$,

ii) $P_{\omega}$ is bounded in $L^{p}\left(\mathbb{R}^{n}\right)$,

iii) $\omega \in R H_{p}^{d}\left(\mathbb{R}^{n}\right)$,

iv) $M_{\omega}$ is bounded in $L^{q}\left(\mathbb{R}^{n}\right)$,

v) $S_{\omega}$ is bounded in $L^{q}\left(\mathbb{R}^{n}\right)$,

where $M_{\omega}$ and $S_{\omega}$ are weighted maximal and square functions, namely, for $E_{j}^{\omega} f=E_{j} f \omega / E_{j} \omega$, and $\Delta_{j}^{\omega}=E_{j+1}^{\omega}-E_{j}^{\omega}$, let

$$
M_{\omega} f=\sup _{j} E_{j}^{\omega}|f| \quad \text { and } \quad S_{\omega} f=\left(\sum_{j}\left|\Delta_{j}^{\omega} f\right|^{2}\right)^{1 / 2},
$$

and $\omega \in R H_{p}^{d}\left(\mathbb{R}^{n}\right)$ (dyadic reverse Hölder $p$ ) means that for each dyadic cube $Q$,

$$
\frac{1}{|Q|} \int_{Q} \omega^{p}(x) d x \leq C\left(\frac{1}{|Q|} \int_{Q} \omega\right)^{p}
$$

Notice that there are doubling weights in some $R H_{p}^{d}$ classes that are not in all of them. For those weights, $P_{\omega}$ and $T_{\omega}$ are bounded in some $L^{p}$ spaces but not in all of them. Therefore they are not dyadic Calderón-Zygmund operators (C-Z).

Operating formally with $P_{\omega}$ we get the Neumann series for $I-\Pi_{b}$, where $\Pi_{b}$ is the dyadic paraproduct (see [Ch]), and $b \in$ BMO (we do not use the standard correspondence $\omega=e^{b}$ but a different one first used in $[\mathrm{KFP}]$ and fully developed by S. Buckley in his Ph.D. Thesis). 
The $R H_{p}^{d}$ condition on $\omega$ is not enough to imply that the paraproduct is a contraction (if it were, the Neumann series would trivially converge to the inverse of $I-\Pi_{b}$ ), but it does guarantee the convergence of $P_{\omega}$.

Loosely speaking, given a locally integrable function $b$, the weight $\omega$, associated to it under this correspondence, is dyadic doubling if and only if there exists a constant $0<\varepsilon<1$ such that for all $j \in \mathbb{Z}$, $\left|\Delta_{j} b\right| \leq 1-\varepsilon$. We will say that such a function $b$ is of $A_{\infty}^{d}$-type if the weight associated to $b$ under the correspondence mentioned before, is in the Muckenhoup class of weights $A_{\infty}^{d}$ (see [GC-RF]). Similarly for $R H_{p}^{d}$-type. In particular these sets of functions are subsets of dyadic BMO.

There is an operator, denoted by $P_{b}$, that can be identified with $P_{\omega}$, for $\omega$ and $b$ related under this correspondence. We will show that whenever $P_{b}$ is well defined and is a bounded operator, then it is the inverse of $I-\Pi_{b}$.

As a consequence of Theorem I and the previous remarks, we can show that,

Theorem II. Given a locally integrable function $b$ and $0<\varepsilon<1$ such that for all $j \in \mathbb{Z},\left|\Delta_{j} b\right| \leq 1-\varepsilon$. Then the operator $I-\Pi_{b}$ has a bounded inverse in some $L^{p}\left(\mathbb{R}^{n}\right), 1<p<\infty$, if and only if $b$ is of $A_{\infty}^{d}$-type. In that case, $\left(I-\Pi_{b}\right)^{-1}=P_{b}$.

Moreover the necessary and suficient condition for having a bounded inverse in $L^{p}\left(\mathbb{R}^{n}\right)$ is that $b$ is of $R H_{p}^{d}$-type.

Since the paraproduct is a bilinear operator in $b$ and $f$, then the existence of a bounded inverse on $L^{p}\left(\mathbb{R}^{n}\right)$ for $I-\lambda \Pi_{b}$ depends on the function $\lambda b$ satisfying the hypothesis of the previous theorem. If it does then $\mu=1 / \lambda$ belongs to the resolvent of $\Pi_{b}$.

We remark that we are dealing here with operators that are not necessarily compact operators. See $[R]$ for some resuls on compact paraproducts.

By previous observations, although $I-\Pi_{b}$ is a dyadic C-Z operator for $b \in \mathrm{BMO}$, the inverse is not necessarily a dyadic C-Z operator.

The representation $P_{b}$ really goes beyond the contractive case.

Theorem III. Given $-1 \leq \lambda<0$, there exist $1<p<\infty, 0<\varepsilon<1$, and a locally integrable function $b$ such that for all $j \in \mathbb{Z},\left|\Delta_{j} b\right| \leq 1-\varepsilon$ and $b$ is of $R H_{p}^{d}$-type but $\lambda b$ is not of $R H_{p}^{d}$-type. In particular, for that $b, I-\Pi_{b}$ is invertible but $\left\|\Pi_{b}\right\|_{p, p} \geq 1 /|\lambda|$. 
The paper is organized as follows. First we will study the invertibility of $I-\Pi_{b}$, and prove Theorem II up to the boundedness of $P_{b}$. In the second part we will study the operators $P_{\omega}$ and $T_{\omega}$. Finally we will clarify the correspondence $b \leftrightarrow \omega$ and the identification of $P_{b}$ and $P_{\omega}$, and we will produce the non-contractive examples of Theorem III.

These results are, in their first version $n=1, p=2$, part of my Ph.D. Thesis. I would like to thank my advisor, P.W. Jones for suggesting the initial problem and guiding me through the completion of this work. I extend my thanks to R.R. Coifman for very helpful conversations.

\section{Inverting Paraproducts.}

\subsection{Preliminaries.}

\subsubsection{Dyadic grid and Haar basis in $\mathbb{R}^{n}$.}

Throughout this paper, we will use " $C$ " to indicate a constant that depends only on $p$ and the dimension $n . \mathcal{D}=\mathcal{D}\left(\mathbb{R}^{n}\right)$ indicates the set of all dyadic cubes in $\mathbb{R}^{n}$. Denote by $\mathcal{D}_{k}$ the $k^{\text {th }}$ generation of $\mathcal{D}$, consisting of those dyadic cubes with side length $2^{-k}$.

Let us define the $n^{\text {th }}$-dimensional Haar basis.

To each dyadic cube $Q=I_{1} \times \cdots \times I_{n}$ we associate $2^{n}-1$ functions indexed by $A_{n}=\left\{e=\left(e_{1}, \ldots, e_{n}\right): e_{i}=1,0 ; e \neq 0\right\}$. For $\mathbf{x}=$ $\left(x_{1}, \ldots, x_{n}\right)$ we set

$$
\psi_{Q}^{e}(\mathbf{x})=\psi_{I_{1}}^{e_{1}}\left(x_{1}\right) \cdots \psi_{I_{n}}^{e_{n}}\left(x_{n}\right)
$$

where

$$
\psi_{I}^{0}(x)=\frac{1}{|I|^{1 / 2}} \chi_{I}(x) \quad \text { and } \quad \psi_{I}^{1}(x)=h_{I}(x) ;
$$

here $\chi_{I}$ and $h_{I}$ denote respectively the characteristic and the Haar functions associated with the interval $I$; more precisely, $h_{I}$ is $\pm 1 /|I|^{1 / 2}$ depending if you are on the right or the left half of $I$, and zero otherwise.

These $n$-dimensional Haar funtions are a basis of $L^{2}\left(\mathbb{R}^{n}\right)$. 


\subsubsection{Expectation and difference operators.}

Let us define for $k \in \mathbb{Z}$ the operators expectation $E_{k}$, and difference $\Delta_{k}$ by

$$
\begin{gathered}
E_{k} f(x)=\frac{1}{|Q|} \int_{Q} f(t) d t, \quad x \in Q \in \mathcal{D}_{k}, \\
\Delta_{k} f(x)=E_{k+1} f(x)-E_{k} f(x) .
\end{gathered}
$$

Since $E_{k} \rightarrow I$ (here $I$ denotes the identity operator) as $k \rightarrow+\infty$, and $E_{k} \rightarrow 0$ as $k \rightarrow-\infty$, both limits are in the $L^{p}$ sense; then the following equalities hold in the $L^{p}\left(\mathbb{R}^{n}\right)$ sense, $E_{k}=\sum_{j<k} \Delta_{j}$, and $I=$ $\sum_{j \in \mathbb{Z}} \Delta_{j}$

Finally, it is not hard to check that

$$
\Delta_{k} f(x)=\sum_{Q \in \mathcal{D}_{k}} \sum_{e \in A_{n}}\left\langle f, \psi_{Q}^{e}\right\rangle \psi_{Q}^{e}(x)
$$

This proves that the Haar system is complete.

\subsubsection{Paraproducts and BMO.}

Define formally the paraproduct associated to a locally integrable function $b$ by

$$
\Pi_{b} f=\sum_{k \in \mathbb{Z}} E_{k} f \Delta_{k} b .
$$

Let us compute formally its adjoint $\Pi_{b}^{*}\left(\int T f g=\int f T^{*} g, f \in L^{p}\right.$, $\left.g \in L^{q}, 1 / p+1 / q=1\right)$

$$
\Pi_{b}^{*} f=\sum_{k \in \mathbb{Z}} \Delta_{k} f \Delta_{k} b .
$$

A locally integrable function $b$ is in dyadic $\mathrm{BMO}_{d}\left(\mathbb{R}^{n}\right)$ if

$$
\|b\|_{*}=\sup _{Q \in \mathcal{D}} \frac{1}{|Q|} \int_{Q}\left|b(x)-m_{Q} b\right| d x \leq C
$$

where $m_{Q} b=\int_{Q} b /|Q|$ 
A remarkable fact, due to John and Niremberg (see [G, Chapter $\mathrm{VI}]$ ), is that for all $1<p<\infty$,

$$
\|b\|_{*} \sim \sup _{Q \in \mathcal{D}}\left(\frac{1}{|Q|} \int_{Q}\left|b(x)-m_{Q} b\right|^{p} d x\right)^{1 / p} .
$$

If $b \in \mathrm{BMO}_{d}\left(\mathbb{R}^{n}\right)$ then the paraproduct is a bounded operator on $L^{p}\left(\mathbb{R}^{n}\right)$, for a proof see [M, p. 273]. Moreover $\left\|\Pi_{b} f\right\|_{p} \leq C\|b\|_{*}\|f\|_{p}$.

Recall the following lemma,

Carleson's Lemma. Let $\left\{a_{Q}\right\}_{Q \in \mathcal{D}}$ be a sequence of positive numbers, that satisfies a Carleson condition, i.e

$$
\sum_{Q \in \mathcal{D}\left(Q_{0}\right)} a_{Q} \leq C\left|Q_{0}\right|, \quad \text { for all } Q_{0} \in \mathcal{D}
$$

Then for any sequence of positive numbers $\lambda_{Q}, Q \in \mathcal{D}$,

$$
\sum_{Q \in \mathcal{D}} a_{Q} \lambda_{Q} \leq \int \lambda^{*}(x) d x, \quad \text { where } \lambda^{*}(x)=\sup _{x \in Q} \lambda_{Q} .
$$

For a proof see [M, p. 273].

Actually, for our dyadic version of the paraproduct, boundedness in $L^{2}\left(\mathbb{R}^{n}\right)$ is a consequence of Carleson's lemma and the boundedness of the dyadic Hardy-Littlewood maximal operator

$$
M f(x)=\sup _{j \in \mathbb{Z}} E_{j}|f|(x)
$$

in $L^{2}\left(\mathbb{R}^{n}\right)$. Just observe that $b \in \mathrm{BMO}_{d}$ implies that the sequence $\left\{|Q| b_{Q}^{2}\right\}$ is a Carleson sequence (by (1)), for $p=2$ ), where $b_{Q}=$ $\Delta_{j} b\left(x_{Q}\right), Q \in \mathcal{D}_{j+1}$, and $x_{Q} \in Q$. Set $\lambda_{Q}=\left(E_{j} f\left(x_{Q}\right)\right)^{2}, Q$ as before; then $\lambda^{*}(x) \leq M f(x)^{2}$. By Littlewood-Paley theory (see Section 3.1.2), and since by (5), $\Delta_{j}\left(\Pi_{b} f\right)=E_{j} f \Delta_{j} b$, then,

$$
\left\|\Pi_{b} f\right\|_{2}^{2} \sim\left\|\left(\sum_{j}\left|E_{j} f \Delta_{j} b\right|^{2}\right)^{1 / 2}\right\|_{2}^{2} .
$$

This is equal to,

$$
\sum_{j} \sum_{Q \in \mathcal{D}_{j+1}}|Q|\left|\Delta_{j} b\left(x_{Q}\right)\right|^{2}\left|E_{j} f\left(x_{Q}\right)\right|^{2}=\sum_{Q \in \mathcal{D}}|Q| b_{Q}^{2} \lambda_{Q}
$$


The right hand side is bounded by $C \int(M f)^{2}$ by Carleson's lemma, and this in turn is bounded by $C\|f\|_{2}^{2}$.

\subsection{Inverting $I-\Pi_{b}$.}

Let $b \in \mathrm{BMO}_{d}\left(\mathbb{R}^{n}\right)$. Given $g \in L^{p}\left(\mathbb{R}^{n}\right)$ we want to solve the following equation for $f \in L^{p}\left(\mathbb{R}^{n}\right)$,

$$
\left(I-\Pi_{b}\right) f=g .
$$

If the paraproduct is a contraction (i.e. $\left\|\Pi_{b}\right\|_{p, p}<1$ ) we can certainly solve the equation. The solution will be given by the Neumann series of $I-\Pi_{b}$, more precisely,

$$
f=\sum_{n=0}^{\infty} \Pi_{b}^{n} g .
$$

We would like to find a solution that goes beyond the contractive case.

Suppose there exist $f, g \in L^{p}\left(\mathbb{R}^{n}\right)$ satisfying the equation

$$
\left(I-\Pi_{b}\right) f=g .
$$

Then $f=g+\Pi_{b} f$. We can look to its-Haar decomposition at level $m$ and get, with the notation in Section 2.1.2, $\Delta_{m} f=\Delta_{m} g+\Delta_{m}\left(\Pi_{b} f\right)$. Since $\Delta_{m} f=E_{m+1} f-E_{m} f$, and $\Delta_{m}\left(\Pi_{b} f\right)=\Delta_{m} b E_{m} f$, we get the following recurrence equation,

$$
E_{m+1} f=\Delta_{m} g+\left(1+\Delta_{m} b\right) E_{m} f .
$$

Solving this equation we conclude that

$$
E_{m+1} f=\sum_{k<m} \Delta_{k} g \prod_{j=k+1}^{m}\left(1+\Delta_{j} b\right)+\Delta_{m} g .
$$

Since $f \in L^{p}\left(\mathbb{R}^{n}\right)$, the right hand side converges to $f$ in the $L^{p}$ sense, so it does the left hand side.

Define the operators $P_{b}^{m}$ applied to a function $g \in L^{p}\left(\mathbb{R}^{n}\right)$ by the formula on the left hand side of (2). And define $P_{b}$ as the limit (if it 
exists) in the $L^{p}$ sense of the $P_{b}^{m}$, as $m \rightarrow+\infty$. With this in mind, at least formally, we can write the formula,

$$
P_{b} g=\sum_{k \in \mathbb{Z}} \Delta_{k} g \prod_{j=k+1}^{\infty}\left(1+\Delta_{j} b\right) .
$$

Notice that $P_{b}$ will be bounded in $L^{p}\left(\mathbb{R}^{n}\right)$ if the operators $P_{b}^{m}$ are uniformly bounded. By construction if $P_{b} g$ is well defined it must produce a solution $f=P_{b} g$ to the equation $\left(I-\Pi_{b}\right) f=g$. We will prove the following theorem,

Theorem 1. The operator $I-\Pi_{b}$ has a bounded inverse in $L^{p}\left(\mathbb{R}^{n}\right)$ if and only if the operator $P_{b}$ is bounded in $L^{p}\left(\mathbb{R}^{n}\right)$. Moreover in that case

$$
\left(I-\Pi_{b}\right)^{-1}=P_{b} .
$$

Now we want to understand the operator $P_{b}$. We will find necessary and sufficient conditions for its boundedness in $L^{p}\left(\mathbb{R}^{n}\right)$, under some mild smallness assumption on the function $b$.

Theorem 2. Given a locally integrable function $b$ and $0<\varepsilon<1$, such that for all $j \in \mathbb{Z},\left|\Delta_{j} b\right| \leq 1-\varepsilon$, then the operator $P_{b}$ is bounded in $L^{p}\left(\mathbb{R}^{n}\right)$ if and only if the function $b$ is of reverse Hölder $p$ type $\left(R H_{p}^{d}\right.$. type).

The condition on $b$ will be described in Section 4 , for now let us just say that such $b$ needs to belong to $\operatorname{BMO}\left(\mathbb{R}^{n}\right)$. We say that $b$ is of $A_{\infty}^{d}$-type if there exists a $1<p<\infty$ so that $b$ is of $R H_{p}^{d}$-type.

As an immediate consequence of the previous theorems we get

Theorem II. For functions $b$ as before. The operator $I-\Pi_{b}$ has a bounded inverse in $L^{p}\left(\mathbb{R}^{n}\right)$ for some $1<p<\infty$, if and only if $b$ of $A_{\infty}^{d}$-type. In that case $\left(I-\Pi_{b}\right)^{-1}=P_{b}$.

This representation of $I-\Pi_{b}^{-1}$ really goes beyond the contractive case. The representation $P_{b}$ captures cancellation between the terms of the Neumann series that is otherwise neglected.

Theorem III. Given $-1 \leq \lambda<0$, there exist $1<p<\infty, 0<\varepsilon<1$, and a locally integrable function $b$ such that for all $j \in \mathbb{Z},\left|\Delta_{j} b\right| \leq 1-\varepsilon$ 
and $b$ is of $R H_{p}^{d}$-type but $\lambda b$ is not of $R H_{p}^{d}$-type. In particular, for that $b, I-\Pi_{b}$ is invertible but $\left\|\Pi_{b}\right\|_{p, p} \geq 1 /|\lambda|$.

REMARK. For a suitable function $b \in \mathrm{BMO}_{d}\left(\mathbb{R}^{n}\right)$, the operator $I-\Pi_{b}$ provides an example of a dyadic $\mathrm{C}-\mathrm{Z}$ operator (see [Ch]) whose inverse is bounded in some $L^{p_{0}}$ but not in every $L^{p}, 1<p<\infty$, therefore $\left(I-\Pi_{b}\right)^{-1}$ is not a dyadic C-Z operator. To produce such examples it is enough to observe that there exists $b$ of $R H_{p_{0}}^{d}$-type which is not of $R H_{p}^{d}$-type for some other $1<p<\infty$.

Examples like these have been produced by $\mathrm{Ph}$. Tchamitchian, see [M. p. 300].

In the next section we will prove Theorem 1 , in Section 3 we will prove Theorem 2 , and in the last section we will construct the examples for Theorem 3 .

\subsection{Proof of Theorem 1.}

We want to find the inverse of $I-\Pi_{b}$. We can formally write down the power series

$$
\sum_{j=0}^{\infty} \Pi_{b}^{j} g \quad \text { (paraseries) }
$$

This series will certainly converge in $L^{p}\left(\mathbb{R}^{n}\right)$ if the paraproduct is a contraction, i.e. $\left\|\Pi_{b}\right\|_{p, p}<1$. This will happen if $b$ has small $\mathrm{BMO}_{d}\left(\mathbb{R}^{n}\right)$ norm. In this case we can compute the inverse $I-\Pi_{b}^{-1}$ and it will coincide with the paraseries.

Operating formally on $P_{b} g$ we get that $P_{b} g=\sum_{j=0}^{\infty} \Pi_{b}^{j} g$.

We would like to show that whenever $P_{b}$ is bounded in $L^{p}\left(\mathbb{R}^{n}\right)$ then

$$
P_{b} f=\left(I-\Pi_{b}\right)^{-1} f, \quad \text { for all } f \in L^{p}\left(\mathbb{R}^{n}\right) .
$$

The rest of the discussion will concentrate on trying to make this argument rigorous.

It is enough to prove a local version of the result. By local we mean to replace $\mathbb{R}^{n}$ by any fixed dyadic cube $Q_{0}$. Because of the scaling invariance we can choose the unit cube $[0,1]^{n}$. In this case, just consider the Haar functions associated to the dyadic cubes $Q \subset Q_{0}$, and let us assume that the $L^{p}$ functions we are dealing with have mean value zero. 
In this setting the operators

$$
P_{b}^{N} g=\sum_{k=0}^{N-1} \Delta_{k} g \prod_{j=k+1}^{N}\left(1+\Delta_{j} b\right)+\Delta_{N} g,
$$

are well defined for locally integrable functions $g$ and $b$, since they are just finite sums of finite products; hence we are free to operate with them.

\subsection{1. $E_{k}, \Delta_{k}$ algebra.}

Fix $Q_{0}=[0,1]^{n}$ once and for all. Let $\mathcal{D}=\mathcal{D}\left(Q_{0}\right)$ denote the dyadics contained in $Q_{0}$ and let $\mathcal{D}_{k}\left(Q_{0}\right)$ denote the $\mathrm{k}^{\text {th }}$ generation, consisting of those cubes in $Q \in \mathcal{D}\left(Q_{0}\right)$ with sidelength $l(Q)=2^{-k} l\left(Q_{0}\right)$. Let $L_{0}^{p}\left(Q_{0}\right)$ denote the subspace of $L^{p}\left(Q_{0}\right)$ of functions with mean value zero on $Q_{0}\left(\int_{Q_{0}} f=0\right)$.

The expectation and difference operators $E_{k}, \Delta_{k}$, are defined as before except that now $k \geq 0$ and the dyadic cubes involved are those $Q \in \mathcal{D}\left(Q_{0}\right)$. The paraproduct and its adjoint are defined similarly. Just for the record, and remembering that we will be working with functions with mean value zero on the base cube $Q_{0}$, i.e. $E_{0} f=0$, we have $E_{k}=\sum_{j=0}^{k-1} \Delta_{j}$, and

$$
\begin{aligned}
& \Pi_{b} f=\sum_{k=0}^{\infty} E_{k} f \Delta_{k} b, \\
& \Pi_{b}^{*} f=\sum_{k=0}^{\infty} \Delta_{k} f \Delta_{k} b .
\end{aligned}
$$

Notice that in both the local and global versions

$$
\Delta_{k}\left(\Pi_{b} f\right)=E_{k} f \Delta_{k} b .
$$

The following composition and product rules hold (see [Ga] for similar results on more general martingales),

$$
\begin{aligned}
E_{k} \Delta_{j} & =\left\{\begin{array}{cl}
\Delta_{j}, & \text { if } j<k, \\
0, & \text { otherwise, }
\end{array}\right. \\
\Delta_{k} \Delta_{j} & =\left\{\begin{array}{cl}
\Delta_{j}, & \text { if } k=j, \\
0, & \text { otherwise, }
\end{array}\right. \\
E_{k} E_{j} & =E_{\min \{k, j\}}, \\
\Delta_{k} f \times \Delta_{j} g & =\Delta_{k}\left(f \times \Delta_{j} g\right) \quad \text { when } k>j .
\end{aligned}
$$


In particular if $k \leq M$, and $0 \leq i_{1}<i_{2}<\cdots<i_{s}=M$, then

$$
\begin{aligned}
E_{k}\left(\Delta_{i_{1}} f_{1} \times \cdots \times \Delta_{i_{s}} f_{s}\right) & =0 \\
E_{k}\left(\sum_{i \geq M} \Delta_{i}\right) & =0 .
\end{aligned}
$$

The operator $P_{b}$ becomes with this notation

$$
P_{b} g=\sum_{k=0}^{\infty} \Delta_{k} g \prod_{j=k+1}^{\infty}\left(1+\Delta_{j} b\right) .
$$

\subsubsection{Theorem 1 - Local version.}

Let $f_{N}=\sum_{k=0}^{N} \Delta_{k} f$ (projection onto low frequency space). Recall that in this setting, we define

$$
P_{b}^{N} g=\sum_{k=0}^{N-1} \Delta_{k} g \prod_{j=k+1}^{N}\left(1+\Delta_{j} b\right)+\Delta_{N} g
$$

For $g \in L_{0}^{p}\left(Q_{0}\right)$, we define $P_{b} g$ as the limit (if it exists) as $N \rightarrow \infty$ in the $L^{p}$ sense of $P_{b}^{N} g$.

Then clearly $P_{b}^{N} g=P_{b_{N}} g_{N}$. The main formula says that on the subspace of step functions $f=f_{N}$, the operator $P_{b_{N}}$ is the inverse of the operator $I-\Pi_{b_{N}}$.

Lemma 1 (Main formula). If $g$ has mean value zero on $Q_{0}$ then for all positive integers $N$,

$$
P_{b_{N}}\left(I-\Pi_{b_{N}}\right) g_{N}=\left(I-\Pi_{b_{N}}\right) P_{b_{N}} g_{N}=g_{N} .
$$

This last statement is a purely algebraic issue. We will prove it later. Let us assume it is true for a moment.

If we consider step functions $b_{N}$, clearly when the operator $P_{b_{N}}$ acts on frequencies higher than $N$, it behaves like the identity. In particular $P_{b_{N}}\left(g-g_{N}\right)=g-g_{N}$.

Notice also that by (8), the paraproduct associated to $b_{N}$ annihilates frequencies higher than $N$; in particular, $\Pi_{b_{N}}\left(g-g_{N}\right)=$ $\sum_{k=0}^{N} \Delta_{k} b E_{k}\left(g-g_{N}\right)=0$. 
After these remarks and using the main formula, we conclude that $\left(I-\Pi_{b_{N}}\right) P_{b_{N}} g=P_{b_{N}}\left(I-\Pi_{b_{N}}\right) g=g$, for all $g \in L_{0}^{p}\left(Q_{0}\right)$.

So we found that on $L_{0}^{p}\left(Q_{0}\right)$ then

$$
P_{b_{N}}=\left(I-\Pi_{b_{N}}\right)^{-1}, \quad \text { for all } N \geq 0 .
$$

With a limiting argument we can show

Theorem 1'. The operator $I-\Pi_{b}$ has a bounded inverse in $L_{0}^{p}\left(Q_{0}\right)$ if and only if $P_{b}$ is a bounded operator on $L^{p}\left(Q_{0}\right)$. Moreover $P_{b}=$ $\left(I-\Pi_{b}\right)^{-1}$ on $L_{0}^{p}\left(Q_{0}\right)$

Proof. It is clear that if there exists a bounded inverse it will have to coincide with $P_{b}$, by construction. We have to prove that if $P_{b}$ is bounded then there is a bounded inverse and they coincide.

It is enough to show that

$$
\int g f=\int P_{b}\left(I-\Pi_{b}\right) g f=\int\left(I-\Pi_{b}\right) P_{b} g f,
$$

for all $f \in L^{q}\left(Q_{0}\right), g \in L_{0}^{p}\left(Q_{0}\right)$.

Recall that $E_{M+1} f=f_{M}$. Assume $M<N$ and let $T^{*}$ denote the adjoint of $T$. Recall that $P_{b_{N}} g=P_{b_{N}} g_{N}+\left(g-g_{N}\right)$.

Notice that by the product and composition rules (6), $P_{b_{N}} g_{N}=$ $E_{N+1}\left(P_{b} g\right)$. Also by (4) $\Pi_{b_{N}}^{*} f_{M}=\Pi_{b}^{*} f_{M}$, for all $M \leq N$. Then using these equalities and (10) we get that, for $M \leq N$,

$$
\begin{aligned}
\int g f_{M} & =\int\left(I-\Pi_{b_{N}}\right) P_{b_{N}} g f_{M} \\
& =\int P_{b_{N}} g\left(I-\Pi_{b_{N}}^{*}\right) f_{M} \\
& =\int\left(P_{b_{N}} g_{N}+g-g_{N}\right)\left(I-\Pi_{b}^{*}\right) f_{M} \\
& =\int E_{N+1}\left(P_{b}(g)\right)\left(I-\Pi_{b}^{*}\right) f_{M}+\int\left(g-g_{N}\right)\left(I-\Pi_{b}^{*}\right) f_{M} .
\end{aligned}
$$

Clearly the left hand side of the string of equalities converges to $\int g f$, for all $f \in L^{q}\left(Q_{0}\right), g \in L_{0}^{p}\left(Q_{0}\right)$ when $N$ and $M \rightarrow \infty$.

Fix $M$ and let $N \rightarrow \infty$. Since $\Pi_{b}^{*}$ is a bounded operator on $L^{q}\left(Q_{0}\right)$ and $\left\|g-g_{N}\right\|_{p} \rightarrow 0$ as $N \rightarrow \infty$, the second term in the last equality 
goes to zero by Hölder's inequality. Since $P_{b}$ is, by hypothesis, bounded on $L^{p}\left(Q_{0}\right)$ we see that, as $N \rightarrow \infty$, the first term converges to

$$
\int P_{b} g\left(I-\Pi_{b}^{*}\right) f_{M}=\int\left(I-\Pi_{b}\right) P_{b} g f_{M}
$$

by the dominated convergence theorem.

Finally let $M \rightarrow \infty$ and again by dominated convergence we see that

$$
\int f g=\int\left(I-\Pi_{b}\right) P_{b} g f, \quad \text { for all } f \in L^{q}\left(Q_{0}\right), g \in L_{0}^{p}\left(Q_{0}\right) .
$$

Similarly for $P_{b}\left(I-\Pi_{b}\right)$. This ends the proof of the theorem.

\subsubsection{Proof of the main formula.}

Fix $N$, and let $b_{N}=\sum_{j=0}^{N} \Delta_{j} b$.

We want to prove that the operator $P_{b_{N}}$ is the inverse of $I-\Pi_{b_{N}}$ on the finite dimensional subspace of functions of frequencies lower or equal to $N$ and mean value zero on $Q_{0}$, namely

$$
L_{0}^{N}=\left\{g \in L_{\mathrm{loc}}^{1}\left(Q_{0}\right): g=\sum_{j=0}^{N} \Delta_{j} g, E_{0} g=0\right\}
$$

It is enough to prove one of the equalities in the lemma, the other is given automatically since we are dealing with a finite dimensional subspace.

We defined

$$
P_{b}^{m} g=\sum_{k=0}^{m-1} \Delta_{k} g \prod_{j=k+1}^{m}\left(1+\Delta_{j} b\right)+\Delta_{m} g .
$$

It follows immediatly from the definition, that the operators $P_{b}^{m}$ satisfy the following recurrence equation

$$
P_{b}^{m} g=\Delta_{m} g+\left(1+\Delta_{m} b\right) P_{b}^{m-1} g .
$$

Let $g \in L_{0}^{N}\left(Q_{0}\right)$, hence $g=g_{N}=\sum_{k=0}^{N} \Delta_{k} g$. Clearly $P_{b}^{N} g=P_{b_{N}} g_{N}$. It is not hard to check that for $m \leq N, P_{b_{N}}^{m} g_{N}=E_{m+1}\left(P_{b_{N}} g_{N}\right)$ and $\Delta_{m} b=\Delta_{m} b_{N}$. Substituting into (11) we get, for $m \leq N$,

$$
E_{m+1}\left(P_{b_{N}} g_{N}\right)=\Delta_{m} g+\left(1+\Delta_{m} b_{N}\right) E_{m}\left(P_{b_{N}} g_{N}\right),
$$


which is the the same recurrence equation we obtained when we solved formally the equation $\left(I-\Pi_{b}\right) f=g$, in Section 2.2.

Recall that by definition of the paraproduct, $\Delta_{k}\left(\Pi_{b} f\right)=\Delta_{k} b E_{k} f$ (see (5)). Also recall that $\Delta_{k}=E_{k+1}-E_{k}$. With this in mind, equation (12) becomes

$$
\Delta_{m}\left(\left(I-\Pi_{b_{N}}\right) P_{b_{N}} g_{N}\right)=\Delta_{m} g .
$$

This is true for all positive integers $m \leq N$. Recall that $E_{N+1}=$ $\sum_{m=0}^{N} \Delta_{m}$, hence we conclude that

$$
E_{N+1}\left(\left(I-\Pi_{b_{N}}\right) P_{b_{N}} g_{N}\right)=E_{N+1} g .
$$

We are almost done; just recall that by definition $E_{N+1} g=g_{N}$, and it is not hard to chek that $E_{N+1}\left(\left(I-\Pi_{b_{N}}\right) P_{b_{N}} g_{N}\right)=\left(I-\Pi_{b_{N}}\right) P_{b_{N}} g_{N}$.

Hence we showed that

$$
\left(I-\Pi_{b_{N}}\right) P_{b_{N}} g_{N}=g_{N}
$$

This finishes the proof of the main formula.

Once the main formula is known, we can show some amusing identities.

Lemma 2. $P_{b_{N}} g_{N}=\sum_{j=0}^{N} \Pi_{b_{N}}^{j} g_{N}$

Proof. By the previous result, it is enough to show that the Neumann polynomial $\sum_{j=0}^{N} \Pi_{b_{N}}^{j} g_{N}$ is the inverse of $I-\Pi_{b_{N}}$ on the subspace $L_{0}^{N}\left(Q_{0}\right)$.

Now certainly

$$
\left(I-\Pi_{b_{N}}\right)\left(\sum_{j=0}^{N} \Pi_{b_{N}}^{j} g_{N}\right)=g_{N}-\Pi_{b_{N}}^{N+1} g_{N} .
$$

We will be done if we show that $\Pi_{b_{N}}^{N+1} g_{N}=0$. We will prove this by induction.

For $N=0$ it is true that $\Pi_{b_{0}} g_{0}=\Delta_{0} b E_{0} g=0$, since $E_{0} g=0$.

Assume that $\Pi_{b_{N-1}}^{N} g_{N-1}=0$. We want to show that it is true for $N+1$. It is enough to show that, for all $k \leq N$,

$$
\Pi_{b_{N}}^{k} g_{N}=\Pi_{b_{N-1}}^{k} g_{N-1}+E_{N}\left(\Pi_{b_{N-1}}^{k-1} g_{N-1}\right) \Delta_{N} b
$$


Suppose for a moment that (13) is true, in particular for $k=N$ we get

$$
\Pi_{b_{N}}^{N} g_{N}=\Pi_{b_{N-1}}^{N} g_{N-1}+E_{N}\left(\Pi_{b_{N-1}}^{N-1} g_{N-1}\right) \Delta_{N} b
$$

But by the inductive hypothesis, $\Pi_{b_{N-1}}^{N} g_{N-1}=0$, hence

$$
\Pi_{b_{N}}^{N+1} g_{N}=\Pi_{b_{N}}\left(E_{N}\left(\Pi_{b_{N-1}}^{N-1} g_{N-1}\right) \Delta_{N} b\right) \text {. }
$$

Finally, just recall that by the product and composition rules of the expectation and difference operators ( $c f$. Section 2.1.2),

$$
\Pi_{b_{N}}\left(\Delta_{N} h\right)=\sum_{k=0}^{N} \Delta_{k} b E_{k}\left(\Delta_{N} h\right)=0
$$

and

$$
E_{N}\left(\Pi_{b_{N-1}}^{N-1} g_{N-1}\right) \Delta_{N} b=\Delta_{N} h,
$$

for certain function $h$. Therefore $\Pi_{b_{N}}^{N+1} g_{N}=0$, and the lemma is proved.

Proof of (13). We will show (13) by induction on $k \leq N$ for fixed $N$.

By the observations made above, the last term in the right hand side of (13) can be written as $\Delta_{N}\left(h_{N}^{k}\right)$, for some function $h_{N}^{k}$, and moreover $\Pi_{b_{N}}\left(\Delta_{N}\left(h_{N}^{k}\right)\right)=0$. Hence when applying the paraproduct $\Pi_{b_{N}}$ to (13) we get

$$
\Pi_{b_{N}}^{k+1} g_{N}=\Pi_{b_{N}}\left(\Pi_{b_{N-1}}^{k} g_{N-1}\right)
$$

Using the linear property on $b$ of $\Pi_{b}$, we get that the right hand side is equal to

$$
\Pi_{b_{N-1}}^{k+1} g_{N-1}+\Pi_{b_{N}-b_{N-1}}\left(\Pi_{b_{N-1}}^{k} g_{N-1}\right) \text {. }
$$

By the definition of the paraproduct and since $\Pi_{b_{N}-b_{N-1}} f=\Delta_{N} b E_{N} f$, then

$$
\Pi_{b_{N}}^{k+1} g_{N}=\Pi_{b_{N-1}}^{k+1} g_{N-1}+\Delta_{N} b E_{N}\left(\Pi_{b_{N-1}}^{k} g_{N-1}\right) .
$$

This proves (13) for $k+1$ assuming that it is known for $k$. The only missing step is to check the equation for $k=1$.

Notice that by the bilinear properties of the paraproduct, then

$$
\Pi_{b_{N}} g_{N}=\Pi_{b_{N-1}} g_{N-1}+\Pi_{b_{N}}\left(g_{N}-g_{N-1}\right)+\Pi_{b_{N}-b_{N-1}}\left(g_{N-1}\right) \text {. }
$$


By previous observations $\Pi_{b_{N}-b_{N-1}}\left(g_{N-1}\right)=\Delta_{N} b E_{N}\left(g_{N-1}\right)$, and $\Pi_{b_{N}}\left(g_{N}-g_{N-1}\right)=0$, hence

$$
\Pi_{b_{N}} g_{N}=\Pi_{b_{N-1}} g_{N-1}+\Delta_{N} b E_{N}\left(g_{N-1}\right)
$$

which is exactly what we wanted to show, since $\Pi_{b_{N-1}}^{0}$ is the identity operator.

We can also show that

Lemma 3. $\Pi_{b_{N}}^{N} g_{N}=\Delta_{0} g \Delta_{1} b \cdots \Delta_{N} b$.

REMARK. From this lemma and property (7) we conclude that

$$
\Pi_{b_{N}}^{N+1} g_{N}=\sum_{k=0}^{N} \Delta_{k} b E_{k}\left(\Delta_{0} g \Delta_{1} b \cdots \Delta_{N} b\right)=0 .
$$

Proof. We know that equation (13) is valid for $k=N-1$, i.e.

$$
\Pi_{b_{N}}^{N-1} g_{N}=\Pi_{b_{N-1}}^{N-1} g_{N-1}+\Delta_{N} b E_{N}\left(\Pi_{b_{N-1}}^{N-2} g_{N-1}\right) .
$$

We will proceed once more by induction. For $N=1$ it is true that $\Pi_{b_{1}} g_{1}=\Delta_{0} b E_{0} g+\Delta_{1} b E_{1} g$, but $E_{0} g=0$ hence $\Delta_{0} g=E_{1} g$, and therefore $\Pi_{b_{1}} g_{1}=\Delta_{0} g \Delta_{1} b$.

Assume that $\Pi_{b_{N-1}^{N-1}}^{N-1} g_{N-1}=\Delta_{0} g \Delta_{1} b \cdots \Delta_{N-1} b$. We want to show that the same holds for $N$. Recall that $\Pi_{b_{N}}\left(\Delta_{N} h\right)=0$, hence, using (14) and the inductive hypothesis, we get that

$$
\begin{aligned}
\Pi_{b_{N}}^{N} g_{N} & =\Pi_{b_{N}}\left(\Delta_{0} g \Delta_{1} b \cdots \Delta_{N-1} b\right) \\
& =\sum_{k=0}^{N} E_{k}\left(\Delta_{0} g \Delta_{1} b \cdots \Delta_{N-1} b\right) \Delta_{k} b
\end{aligned}
$$

the last equality by the definition of the paraproduct. By property (7), we see that all the summands in (15) are cancelled except the last one, more precisely, $E_{k}\left(\Delta_{0} g \Delta_{1} b \cdots \Delta_{N-1} b\right)=0$ for all $0 \leq k<N$, and $E_{N}\left(\Delta_{0} g \Delta_{1} b \cdots \Delta_{N-1} b\right)=\Delta_{0} g \Delta_{1} b \cdots \Delta_{N-1} b$. Hence

$$
\Pi_{b_{N}}^{N} g_{N}=\Delta_{0} g \Delta_{1} b \cdots \Delta_{N-1} b \Delta_{k} b .
$$

This finishes the proof of the lemma. 


\section{Boundedness of $P_{b}$, or $T_{\omega}$ vs $P_{\omega}$.}

In Section 2.2 we introduced the operator $P_{b}$, associated to a given function $b \in \mathrm{BMO}$. Formally the operator is

$$
P_{b} g=\sum_{k \in \mathbb{Z}} \Delta_{k} g \prod_{j=k+1}^{\infty}\left(1+\Delta_{j} b\right) .
$$

We are multiplying the Haar decomposition at level $j$ of $g, \Delta_{j} g$, by an infinite product. Suppose that for each $j$, the product is a function. Then $P_{b}$ will be a particular case of the model operator

$$
T f(x)=\sum_{k \in \mathbb{Z}} \omega_{k}(x) \Delta_{k} f(x),
$$

where the $\omega_{k}$ 's are a sequence of functions. For example, if $\omega_{k}=1$ then $T$ will be the identity operator.

Let us consider the following non trivial examples. Fix $\omega$, a positive locally integrable function (a weight); consider

1) $\omega_{k}^{\prime}(x)=\frac{\omega(x)}{E_{k} \omega(x)}$,

2) $\omega_{k}^{\prime \prime}(x)=\frac{\omega(x)}{E_{k+1} \omega(x)}$,

and call $T_{\omega}$ and $P_{\omega}$ the operators associated to the first and second examples respectively.

In Section 4 we will see that there is a very nice correspondence between some classes of weights $\omega$ and functions $b$ in BMO. Under this correspondence

$$
P_{b}=P_{\omega} .
$$

Therefore it is enough to study $P_{\omega}$.

Suppose $T_{\omega}$ is a bounded operator in $L^{p}\left(\mathbb{R}^{n}\right)$. It can be checked with no difficulty (computing the action of $T_{\omega}$ on Haar functions), that the weight $\omega$ must satisfy the so called dyadic reverse Hölder $p$ condition ( $R H_{p}^{d}$ condition, see definition in the next section).

We will prove the following theorem,

Theorem 4 (Theorem I). Given a dyadic doubling weight $\omega$ (defined in the next Section), for $1<p<\infty$ and $1 / p+1 / q=1$, the following properties are equivalent, 
i) $T_{\omega}$ is bounded in $L^{p}\left(\mathbb{R}^{n}\right)$,

ii) $P_{\omega}$ is bounded in $L^{p}\left(\mathbb{R}^{n}\right)$,

iii) $\omega \in R H_{p}^{d}\left(\mathbb{R}^{n}\right)$,

iv) $M_{\omega}$ is bounded in $L^{q}\left(\mathbb{R}^{n}\right)$,

v) $S_{\omega}$ is bounded in $L^{q}\left(\mathbb{R}^{n}\right)$,

where $M_{\omega}$ and $S_{\omega}$ are weighted maximal and square functions, namely, $M_{\omega} f=\sup _{j} E_{j}^{\omega}|f|$, and $S_{\omega} f=\left(\sum_{j}\left|\Delta_{j}^{\omega} f\right|^{2}\right)^{1 / 2}$, where $E_{j}^{\omega} f=E_{j} f \omega /$ $E_{j} \omega$, and $\Delta_{j}^{\omega}=E_{j+1}^{\omega}-E_{j}^{\omega}$.

This will be enough to prove Theorem 2 , once the relation $\omega \leftrightarrow b$ is understood. Under this correspondence, a weight $\omega$ is dyadic doubling if and only if there exists a constant $0<\varepsilon<1$ such that $\left|\Delta_{j} b\right| \leq 1-\varepsilon$ for all $j \in \mathbb{Z}$. The condition $b$ is of $R H_{p}^{d}$-type means that the corresponding weight $\omega \in R H_{p}^{d}$.

We could also give sufficient conditions for the model operator (17) to be bounded, when a larger class of weights is considered. That can be found in $[\mathrm{P}]$ for $n=1, p=2$.

The proof of the theorem reduces to the boundedness of some weighted dyadic square functions, which in turn are controlled by a weighted maximal function. These are well known objects. We will present a proof, identical to S. Buckley's proof of the boundedness of the standard dyadic square function on weighted $L^{2}$. Our proof will allow enough weights in the game so that not only we capture our $L^{2}$ result, but also we have enough room to use a standard extrapolation argument to produce the $L^{p}$ results, following J. L. Rubio de Francia's philosophy that "there is no $L^{p}$ but weighted $L^{2}$ ".

Once the boundedness on $L^{p}$ of the weighted square functions is established, we get our results using classical Littlewood-Paley theory.

First we will recall some definitions and standard results. Then we will prove Theorem 4 , and finally we will clarify the relation between $\omega$ and $b$, and produce the examples of Theorem 3 . 


\subsection{Preliminaries.}

\subsubsection{Weights.}

Recall that throughout this paper, we are using " $C$ " to indicate a constant that depends only on $p$ and the dimension $n . \mathcal{D}=\mathcal{D}\left(\mathbb{R}^{n}\right)$ indicates the set of all dyadic cubes in $\mathbb{R}^{n}$. For any $Q \in \mathcal{D}, \mathcal{D}(Q)$ is the collection of proper dyadic subcubes of $Q, \tilde{Q}$ is the parent of $Q$ (the smallest dyadic cube properly containing $Q$ ). For any weight $\omega$ and set $S, \omega(S)$ denotes the integral of $\omega$ over $S,|S|$ denotes the Lebesgue measure of $S$, and $m_{S} \omega=\omega(S) /|S|$. Unless otherwise specified, $1<$ $p<\infty$, but $p$ is otherwise arbitrary.

Definition. We say that $\omega$ is a dyadic doubling weight, if there exists a constant $C>0$ such that $\omega(\tilde{Q}) \leq C \omega(Q)$ for all dyadic cubes $Q, \tilde{Q}$ is the parent of $Q$.

Definition. We say that $\omega$ is an $A_{p}^{d}$ weight if there exists $C>0$ such that

$$
\left(\frac{1}{|Q|} \int_{Q} \omega\right)\left(\frac{1}{|Q|} \int_{Q} \omega^{-1 / p-1}\right)^{p-1} \leq C, \quad \text { for all } Q \in \mathcal{D}
$$

Definition. We say that $\omega$ is an $A_{\infty}^{d}$ weight if there exists $C>0$, such that for all $Q \in \mathcal{D}$, we have

$$
\frac{1}{|Q|} \int_{Q} \omega \leq C \exp \left(\frac{1}{|Q|} \int_{Q} \log \omega\right)
$$

Definition. We say that $\omega \in R H_{p}^{d}$ (dyadic reverse Hölder $p$ ) if there exists $C>0$ such that

$$
\frac{1}{|Q|} \int_{Q} \omega^{p}(x) d x \leq C\left(\frac{1}{|Q|} \int_{Q} \omega(x) d x\right)^{p}, \quad \text { for all } Q \in \mathcal{D}
$$

These are dyadic versions of the classical Muckenhoupt classes. For equivalent definitions of weight classes see [GC-RF] and [CF].

It is clear how to define weight classes with respect to a positive measure $\sigma$. 
We will assume that all our weights are dyadic doubling, and we will consistently ommit the word dyadic. This is necessary for the theory of such weights to closely mirror the non-dyadic case, for example to get that $\omega \in R H_{p}^{d}$ implies that $\omega \cdot \in A_{\infty}^{d}$. S. Buckley studied a characterization of these classes of weights by summation conditions, [B]. In particular, given a doubling weight $\omega$,

(18) $\omega \in A_{\infty}^{d}$ if and only if $\sum_{Q \in \mathcal{D}\left(Q_{0}\right)}|Q|\left(\frac{m_{Q} \omega-m_{\tilde{Q}} \omega}{m_{\tilde{Q}} \omega}\right)^{2} \leq C\left|Q_{0}\right|$.

This is a Carleson condition.

Definition. We will say that a sequence of numbers, $\left\{a_{Q}\right\}_{Q \in \mathcal{D}}$, is a $\mu$-Carleson sequence, for a given positive measure $\mu$, if for all $Q_{0} \in \mathcal{D}$,

$$
\sum_{Q \in \mathcal{D}\left(Q_{0}\right)} \mu(Q) a_{Q}^{2} \leq \mu\left(Q_{0}\right)
$$

It is clear what is a doubling measure.

Definition. Given a doubling measure $\sigma$, we will say that the weight $\omega$ is in $A_{\infty}^{d}(d \sigma)$, if the sequence $a_{Q}=\left(m_{Q}^{\sigma} \omega-m_{\tilde{Q}}^{\sigma} \omega\right) / m_{\tilde{Q}}^{\sigma} \omega$ is a $\sigma$ Carleson sequence, where $m_{Q}^{\sigma} \omega=\int_{Q}(\omega / \sigma(Q)) d \sigma$.

Definition. Given a doubling measure $\sigma$, we will say that a weight $\omega$ is $\sigma$-doubling if the measure $d \mu=\omega d \sigma$ is doubling.

We will say that a measure $\mu$ is in $A_{\infty}^{d}(d \sigma)$ if $d \mu=\omega d \sigma$, and $\omega$ is an $A_{\infty}^{d}(d \sigma)$ weight.

We will need the following lemmas

Lemma 4. Given a doubling measure $\sigma$ in $A_{\infty}^{d}(d x)$, and a doubling measure $\mu \in A_{\infty}^{d}(d \sigma)$, then any $\sigma$-Carleson sequence is also $\mu$-Carleson.

Lemma 5. Given a doubling measure $\sigma$ in $A_{\infty}^{d}(d x)$, and a doubling weight $\mu \in A_{\infty}^{d}(d \sigma)$. Then the sequence $\left(m_{Q} \mu-m_{\tilde{Q}} \mu\right) / m_{\tilde{Q}} \mu$ is a $\mu$ Carleson sequence. 
We will prove these lemmas at the end. We will also need a weighted version of Carleson's lemma.

Lemma 6 (Weighted Carleson's lemma). Given a doubling measure $\mu$ in $A_{\infty}^{d}(d x)$. Given a $\mu$-Carleson sequence $\left\{a_{Q}\right\}_{Q \in \mathcal{D}}$, and a sequence of positive numbers $\left\{\lambda_{Q}\right\}_{Q \in \mathcal{D}}$ then,

$$
\sum_{Q \in \mathcal{D}} \mu(Q) a_{Q}^{2} \lambda_{Q} \leq C \int \lambda^{*}(x) d \mu(x)
$$

where $\lambda^{*}(x)=\sup _{x \in Q} \lambda_{Q}$.

For a proof see [M, p. 273], with the obvious changes.

In particular if $\lambda_{Q}=\left(m_{Q}^{\sigma} f\right)^{2}$ then we can bound the sum of the products by the $L^{2}(d \mu)$ norm of the weighted maximal function $M_{\sigma}$ to be defined in the next section.

\subsubsection{Littlewood-Paley theory.}

We will use the notation $a \sim b$, for positive numbers $a$ and $b$, whenever there exists a positive and finite constant $C$ such that $C^{-1} b \leq$ $a \leq C b$; we will say, in that case, that $a$ and $b$ are comparable.

The expectation and difference operators $E_{j}, \Delta_{j}$ where introduced in Section 2.1.2.

The dyadic maximal and square functions are,

$$
M f(x)=\sup _{j} E_{j}|f|(x),
$$

and

$$
S f(x)=\left(\sum_{j}\left|\Delta_{j} f(x)\right|^{2}\right)^{1 / 2}
$$

respectively. It is well known that (see $[\mathrm{Ch}],[\mathrm{Ga}]$ or $[\mathrm{S}]$ )

Theorem 5 (Littlewood-Paley). $\|S f\|_{L^{p}(d x)} \sim\|f\|_{L^{p}(d x)}$. 
We will be interested in weighted versions of the square and maximal functions. Given a doubling measure $\sigma$, define the weighted expectation and difference operators by

$$
\begin{gathered}
E_{j}^{\sigma} f(x)=\frac{1}{\sigma(Q)} \int_{Q} f d \sigma, \quad x \in Q \in \mathcal{D}_{j} \\
\Delta_{j}^{\sigma}=E_{j+1}^{\sigma}-E_{j}^{\sigma} .
\end{gathered}
$$

Define the corresponding maximal and square functions, $M_{\sigma}$ and $S_{\sigma}$

$$
\begin{gathered}
M_{\sigma} f(x)=\sup _{j} E_{j}^{\sigma}|f|(x), \\
S_{\sigma} f(x)=\left(\sum_{j}\left|\Delta_{j}^{\sigma} f(x)\right|^{2}\right)^{1 / 2} .
\end{gathered}
$$

It is certainly true, for doubling measures $\sigma \in A_{\infty}^{d}$, that the $L^{p}(d \sigma)$ norms of the weighted square function and the function are comparable, i.e. $\left\|S_{\sigma} f\right\|_{L^{p}(d \sigma)} \sim\|f\|_{L^{p}(d \sigma)}$, see $[\mathrm{Ga}]$.

The following classical results are known.

Theorem 6. (Coifman-Fefferman). Given a doubling measure $\sigma \in A_{\infty}^{d}$, and a $\sigma$-doubling weight $\omega_{0}$, then $M_{\sigma}$ is bounded in $L^{p}\left(\omega_{0} d \sigma\right)$ if and only if $\omega_{0} \in A_{p}^{d}(d \sigma)$.

For a proof see $[\mathrm{CF}]$ with the obvious changes. It is also true that

Theorem 7. Given a doubling measure $\sigma \in A_{\infty}^{d}$, and a $\sigma$-doubling weight $\omega_{0}$, then $\omega_{0} \in A_{p}^{d}(d \sigma)$ if and only if $S_{\sigma}$ is bounded in $L^{p}\left(\omega_{0} d \sigma\right)$.

We will present a proof of this theorem at the end for completeness. This is an extension of the results of S. Buckley, who proves it for $p=2$ and $d \sigma=d x$ (see [B]). Extrapolation will then give the result for $1<p<\infty$.

Theorem 8 (Rubio de Francia's extrapolation). Given a doubling measure $\sigma \in A_{\infty}^{d}$, and a $\sigma$-doubling weight $\omega_{0}$, if $\omega_{0} \in A_{2}^{d}(d \sigma)$ implies that $T$ is bounded in $L^{2}\left(\omega_{0} d \sigma\right)$ then $\omega_{0} \in A_{p}^{d}(d \sigma)$ implies that $T$ is bounded in $L^{p}\left(\omega_{0} d \sigma\right)$, for all $1<p<\infty$. 
For a proof see [GC-RF].

Let us just mention the following tautology:

$$
\omega_{0} \in A_{p}^{d}(d \sigma) \text { if and only if } \frac{1}{\omega_{0}} \in R H_{q}^{d}\left(\omega_{0} d \sigma\right), \frac{1}{q}+\frac{1}{p}=1 .
$$

In particular, given $\omega$ a doubling weight, let $\omega_{0}=1 / \omega, d \sigma=\omega d x$; then $\omega_{0} d \sigma=d x$. Denote by $S_{\omega}=S_{\sigma}$, then using the tautology, the theorem for the square function reads,

$$
S_{\omega} \text { is bounded in } L^{p}(d x) \text { if and only if } \omega \in R H_{q}^{d}(d x),
$$

which is the equivalence between v) and iii) in Theorem I.

Similarly, denoting by $M_{\omega}=M_{\sigma}$, Theorem 6 for the maximal function reads,

$$
M_{\omega} \text { is bounded in } L^{p}(d x) \text { if and only if } \omega \in R H_{q}^{d}(d x),
$$

which is the equivalence of iv) and iii) in Theorem I.

\section{2. $P_{\omega}$ vs $T_{\omega}$ - Proof of Theorem I.}

After the remarks at the end of previous section, only the equivalence of i), ii) and iii) are left in the proof of Theorem I.

Given a doubling weight $\omega$, define at least formally the operators

$$
\begin{gathered}
P_{\omega} f(x)=\sum_{j} \frac{\omega(x)}{E_{j+1} \omega(x)} \Delta_{j} f(x), \\
T_{\omega} f(x)=\sum_{j} \frac{\omega(x)}{E_{j} \omega(x)} \Delta_{j} f(x) .
\end{gathered}
$$

As we pointed out before, if $T_{\omega}$ is bounded in $L^{p}\left(\mathbb{R}^{n}\right)$ then $\omega \in R H_{p}^{d}\left(\mathbb{R}^{n}\right)$. Computing formally the adjoints with respect to the ordinary pairing, we get that

$$
\begin{gathered}
P_{\omega}^{*} f=\sum_{j} \Delta_{j}\left(\frac{\omega f}{E_{j+1} \omega}\right), \\
T_{\omega}^{*} f=\sum_{j} \Delta_{j}\left(\frac{\omega f}{E_{j} \omega}\right) .
\end{gathered}
$$


Certainly if we can prove that these "adjoints" are well defined and bounded operators in $L^{q}\left(\mathbb{R}^{n}\right)$, then the operators $T_{\omega}$ and $P_{\omega}$ will be well defined themselves and bounded in $L^{p}\left(\mathbb{R}^{n}\right)$.

These suggest us to introduce the following cousins of the weighted square function $S_{\sigma}$, defined in the preliminaries. Define

$$
\begin{aligned}
& S_{\sigma}^{\prime} f(x)=\left(\sum_{j}\left|\Delta_{j}\left(\frac{\sigma f}{E_{j+1} \sigma}\right)(x)\right|^{2}\right)^{1 / 2}, \\
& S_{\sigma}^{\prime \prime} f(x)=\left(\sum_{j}\left|\Delta_{j}\left(\frac{\sigma f}{E_{j} \sigma}\right)(x)\right|^{2}\right)^{1 / 2} .
\end{aligned}
$$

Proposition 1. Given a doubling measure $\sigma \in A_{\infty}^{d}(d x)$, and a doubling measure $\mu \in A_{2}^{d}(d \sigma)$, then $S_{\sigma}^{\prime}$ and $S_{\sigma}^{\prime \prime}$ are bounded in $L^{2}(d \mu)$.

We will prove it at the end.

If that is the case, then by the Extrapolation Theorem 8, we will conclude that

Corollary 1. Given a doubling measure $\sigma \in A_{\infty}^{d}(d x)$, and a doubling measure $\mu \in A_{q}^{d}(d \sigma)$, then $S_{\sigma}^{\prime}$ and $S_{\sigma}^{\prime \prime}$ are bounded in $L^{q}(d \mu)$.

In particular, let $\omega$ be a doubling weight, let $d \sigma=\omega d x, \omega_{0}=1 / \omega$, and $d \mu=\omega_{0} d \sigma=d x$, assume that $\omega \in R H_{p}^{d}(d x)$ (if and only if $1 / \omega \in$ $A_{q}^{d}(\omega d x)$ ); and let us denote $S_{\omega}^{\prime}=S_{\sigma}^{\prime}, S_{\omega}^{\prime \prime}=S_{\sigma}^{\prime \prime}$, then by Corollary 1 ,

$$
\begin{aligned}
\left\|S_{\omega}^{\prime} f\right\|_{L^{q}(d x)} & \leq C\|f\|_{L^{q}(d x)}, \\
\left\|S_{\omega}^{\prime \prime} f\right\|_{L^{q}(d x)} & \leq C\|f\|_{L^{q}(d x)} .
\end{aligned}
$$

Notice that

$$
\begin{gathered}
\Delta_{j}\left(P_{\omega}^{*} f\right)=\Delta_{j}\left(\frac{f \omega}{E_{j+1} \omega}\right), \\
\Delta_{j}\left(T_{\omega}^{*} f\right)=\Delta_{j}\left(\frac{f \omega}{E_{j} \omega}\right) .
\end{gathered}
$$

Therefore, if $S$ is the standard dyadic square function, $S\left(P_{\omega}^{*} f\right)=S_{\omega}^{\prime} f$ and $S\left(T_{\omega}^{*} f\right)=S_{\omega}^{\prime \prime} f$. And by the Littlewood-Paley theory and (19), if $\omega \in R H_{p}^{d}(d x)$ then

$$
\left\|P_{\omega}^{*} f\right\|_{q} \sim\left\|S\left(P_{\omega}^{*} f\right)\right\|_{q}=\left\|S_{\omega}^{\prime} f\right\|_{q} \leq C\|f\|_{q} .
$$


Similarly $\left\|T_{\omega}^{*} f\right\|_{q} \leq C\|f\|_{q}$.

This implies that $P_{\omega}^{*}$ and $T_{\omega}^{*}$ are bounded operators on $L^{q}(d x)$; so certainly $P_{\omega}$ and $T_{\omega}$ will be bounded on $L^{p}(d x)$, provided $\omega \in R H_{p}^{d}(d x)$.

We pointed out before that it is trivial to check that if $T_{\omega}$ is bounded in $L^{p}$ then $\omega \in R H_{p}^{d}(d x)$. For $P_{\omega}$ the same holds; just recall that since $\omega$ is a doubling weight, in particular $E_{j+1} \omega \sim E_{j} \omega$, uniformly on $j$. It is also true that $T_{\omega}$ and $S_{\omega}$ are simultaneously bounded in $L^{p}$.

Therefore we almost proved Thecrem I. After our previous remarks, the only missing step in the proof of this theorem is the proof of the Proposition 1.

Proof of Proposition 1. First note that we can compute the $L^{2}(d \mu)$ norms of the square functions, since

$$
\begin{aligned}
\left\|\left(\sum_{j}\left|\Delta_{j} g\right|^{2}\right)^{1 / 2}\right\|_{L^{2}\left(d_{\mu} \mu\right)}^{2} & =i\left(\sum_{j}\left|E_{j+1} g-E_{j} g\right|^{2}\right)^{1 / 2} \|_{L^{2}(d \mu)}^{2} \\
& =\sum_{Q \in \mathcal{D}} \mu(Q)\left|m_{Q} g-m_{\tilde{Q}} g\right|^{2}
\end{aligned}
$$

where $m_{Q} g=\int_{Q} g /|Q|$.

We will get

$$
\begin{aligned}
\left\|S_{\sigma} f\right\|_{L^{2}(d \mu)}^{2} & =\sum_{Q \in \mathcal{D}} \mu(Q)\left(\frac{m_{Q} f \sigma}{m_{Q} \sigma}-\frac{m_{\tilde{Q}} f \sigma}{m_{\tilde{Q}} \sigma}\right)^{2}, \\
\left\|S_{\sigma}^{\prime} f\right\|_{L^{2}(d \mu)}^{2} & =\sum_{Q \in \mathcal{D}} \mu(Q)\left(\frac{m_{Q} f \sigma}{m_{Q} \sigma}-\frac{1}{2^{n}} \sum_{Q^{\prime} \in S(\tilde{Q})} \frac{m_{Q^{\prime}} f \sigma}{m_{Q^{\prime}} \sigma}\right)^{2}, \\
\left\|S_{\sigma}^{\prime \prime} f\right\|_{L^{2}(d \mu)}^{2} & =\sum_{Q \in \mathcal{D}} \mu(Q)\left(\frac{m_{Q} f \sigma}{m_{\tilde{Q}} \sigma}-\frac{m_{\tilde{Q}} f \sigma}{m_{\tilde{Q}} \sigma}\right)^{2},
\end{aligned}
$$

where $m_{Q} \sigma=\sigma(Q) /|Q|$, and $S(\tilde{Q})$ denotes the subset of dyadic cubes which are direct children of $\tilde{Q}$.

Let us first compare $S_{\sigma}$ and $S_{\sigma}^{\prime \prime}$. Adding and subtracting $m_{Q} f \sigma /$ $m_{\tilde{Q}} \sigma$ we get that

$$
\left\|S_{\sigma} f\right\|_{L^{2}(d \mu)}^{2} \leq C\left\|S_{\sigma}^{\prime \prime} f\right\|_{L^{2}(d \mu)}^{2}
$$




$$
\begin{aligned}
& +C \sum_{Q \in \mathcal{D}} \mu(Q)\left(\frac{m_{Q} f \sigma}{m_{Q} \sigma}\right)^{2}\left(\frac{m_{Q} \sigma-m_{\tilde{Q}} \sigma}{m_{\bar{Q}} \sigma}\right)^{2} \\
= & C\left\|S_{\sigma}^{\prime \prime} f\right\|_{L^{2}(d \mu)}^{2}+C W_{1} .
\end{aligned}
$$

The last summand on the right hand side is bounded by a constant times $\left\|M_{\sigma} f\right\|_{L^{2}(d \mu)}^{2}$. This is a consequence of the weighted Carleson's lemma ( $c f$. Section 3.1.1). Because by Buckley's summation condition (18), and the weight Lemma 4 , the sequence $\left(m_{Q} \sigma-m_{\tilde{Q}} \sigma\right) / m_{\tilde{Q}} \sigma$ is a $\mu$-Carleson sequence, since by hypothesis $\mu \in A_{2}^{d}(d \sigma) \subset A_{\infty}^{d}(d \sigma)$, and $\mu$ is doubling.

Similarly we conclude that

$$
\left\|S_{\sigma}^{\prime \prime} f\right\|_{L^{2}(d \mu)}^{2} \leq C\left\|S_{\sigma} f\right\|_{L^{2}(d \mu)}^{2}+C\left\|M_{\sigma} f\right\|_{L^{2}(d \mu)}^{2} .
$$

Let us now compare $S_{\sigma}^{\prime}$ and $S_{\sigma}$. Adding and subtracting the correct terms, we get

$$
\begin{aligned}
\left\|S_{\sigma}^{\prime} f\right\|_{L^{2}(d \mu)}^{2} \leq & C\left\|S_{\sigma} f\right\|_{L^{2}(d \mu)}^{2} \\
& +C \sum_{Q \in \mathcal{D}} \mu(Q)\left(\frac{m_{\tilde{Q}} f \sigma}{m_{\tilde{Q}} \sigma}-\frac{1}{2^{n}} \sum_{Q^{\prime} \in S(\tilde{Q})} \frac{m_{Q^{\prime}} f \sigma}{m_{Q^{\prime}} \sigma}\right)^{2} .
\end{aligned}
$$

But

$$
m_{\tilde{Q}} f \sigma=\frac{1}{2^{n}} \sum_{Q^{\prime} \in S(\tilde{Q})} m_{Q^{\prime}} f \sigma .
$$

Therefore we can bound the last summand by

$$
S(f, \sigma)=\sum_{Q \in \mathcal{D}} \mu(Q)\left(\frac{1}{2^{n}} \sum_{Q^{\prime} \in S(\tilde{Q})} \frac{m_{Q^{\prime}} f \sigma}{m_{Q^{\prime}} \sigma} \frac{m_{Q^{\prime}} \sigma-m_{\tilde{Q}} \sigma}{m_{\tilde{Q}} \sigma}\right)^{2} .
$$

By hypothesis $\mu$ is doubling, i.e. $\mu(Q) \sim \mu(\tilde{Q})$, for all $Q \in S(\tilde{Q})$; and clearly

$$
S(f, \sigma) \leq C \sum_{Q \in \mathcal{D}} \mu(\tilde{Q})\left(\frac{m_{Q} \sigma-m_{Q} \sigma}{m_{\bar{Q}} \sigma}\right)^{2}\left(\frac{m_{Q} f \sigma}{m_{Q} \sigma}\right)^{2}=W_{1} .
$$

But $W_{1}$ is bounded by a constant multiple of $\left\|M_{\sigma} f\right\|_{L^{2}(d \mu)}^{2}$. We conclude that

$$
\left\|S_{\sigma}^{\prime} f\right\|_{L^{2}(d \mu)}^{2} \leq C\left\|S_{\sigma} f\right\|_{L^{2}(d \mu)}^{2}+C\left\|M_{\sigma} f\right\|_{L^{2}(d \mu)}^{2} .
$$


This finishes the proof of the proposition, since by hypothesis we know that $M_{\sigma}$ is bounded on $L^{2}(d \mu)$ and so is then $S_{\sigma}$ by Theorems 6 and 7 .

Thus, Theorem I has been proved.

\subsection{Proof of the weighted square function theorem d'après Buckley.}

Let us denote the mean value of a function $f$ on a cube $Q$ with respect to the measure $\sigma$ by $m_{Q}^{\sigma} f$. Hence, $m_{Q}^{\sigma} f=m_{Q} f \sigma / m_{Q} \sigma$.

With this notation the weighted square function $S_{\sigma}$ becomes

$$
S_{\sigma} f(x)=\left(\sum_{x \in Q \in \mathcal{D}}\left|m_{Q}^{\sigma} f-m_{\tilde{Q}}^{\sigma} f\right|^{2}\right)^{1 / 2}
$$

Computing the $L^{2}(d \mu)$ norm (see (20)), we get

$$
\left\|S_{\sigma} f\right\|_{L^{2}(d \mu)}^{2}=\sum_{Q \in \mathcal{D}} \mu(Q)\left(m_{Q}^{\sigma} f-m_{\tilde{Q}}^{\sigma} f\right)^{2} .
$$

We want to prove that if $\sigma$ is a doubling weight in $A_{\infty}^{d}(d x)$, and $\omega_{0} \in A_{2}^{d}(d \sigma)$ and is $\sigma$-doubling, then $S_{\sigma}$ is a bounded operator in $L^{2}(d \mu)$, where $d \mu=\omega_{0} d \sigma$.

We know that under those conditions the weighted maximal function $M_{\sigma}$ is bounded in $L^{2}(d \mu)$ (see Theorem 6). Therefore it will be enough to control the square function with the maximal function. More precisely, we will show, exactly as in Buckley's proof for $d \sigma=d x$, $d \mu=\omega d x$, doubling $\omega \in A_{2}^{d}(d x)$ (see [B]), that

$$
\left\|S_{\sigma} f\right\|_{L^{2}(d \mu)}^{2} \leq C\left\|M_{\sigma} f\right\|_{L^{2}(d \mu)}^{2}+C\left\|M_{\sigma} f\right\|_{L^{2}(d \mu)}\left\|S_{\sigma} f\right\|_{L^{2}(d \mu)} .
$$

With a bootstrapping argument, and Theorem 6 we get

$$
\left\|S_{\sigma} f\right\|_{L^{2}(d \mu)}^{2} \leq C\|f\|_{L^{2}(d \mu)}^{2},
$$

provided that we can ensure that the $L^{2}(d \mu)$ norm of $S_{\sigma} f$ is finite.

We will show that (22) holds uniformly for a finite version of the square function. More precisely, fix $N>0$ and $Q_{0}$ any cube. Denote 
by $\mathcal{D}^{N}\left(Q_{0}\right)$ those cubes $Q \in \mathcal{D}\left(Q_{0}\right)$ such that their side length $l(Q) \geq$ $2^{-N} l\left(Q_{0}\right)$. Define

$$
S_{\sigma}^{N, Q_{0}} f(x)=\left(\sum_{x \in Q \in \mathcal{D}^{N}\left(Q_{0}\right)}\left|m_{Q}^{\sigma} f-m_{\tilde{Q}}^{\sigma} f\right|^{2}\right)^{1 / 2} .
$$

Clearly now $\left\|S_{\sigma}^{N, Q_{0}} f\right\|_{L^{2}(d \mu)}<\infty$. Now we are allowed to complete the bootstrapping argument to conclude that

$$
\left\|S_{\sigma}^{N, Q_{0}} f\right\|_{L^{2}(d \mu)}^{2} \leq C\|f\|_{L^{2}(d \mu)}^{2}, \quad \text { for all } N>0 \text {, for all } Q_{0} .
$$

Finally letting $N$ go to infinity, and then adding over the cubes in a given generation, $Q_{0} \in \mathcal{D}_{M}$, and letting $M \rightarrow-\infty$, we will get the desired boundedness of $S_{\sigma}$.

Fix $N>0$ and the base cube $Q_{0}$. To simplify the notation, let us drop the superscripts $N$ and $Q_{0}$ from the operator and the dyadics. Since $\mu$ is a doubling measure, then

$$
\left\|S_{\sigma} f\right\|_{L^{2}(d \mu)}^{2} \leq C \sum_{Q \in \mathcal{D}} \mu(\tilde{Q})\left(m_{Q}^{\sigma} f-m_{\tilde{Q}}^{\sigma} f\right)^{2}=W .
$$

Add and subtract

$$
a_{Q}=\frac{m_{Q} f \sigma}{m_{\tilde{Q}} \sigma}=\frac{m_{Q} \sigma}{m_{\tilde{Q}} \sigma} m_{Q}^{\sigma} f
$$

then,

$$
\begin{aligned}
W \leq & \sum_{Q \in \mathcal{D}} \mu(\tilde{Q})\left(m_{Q}^{\sigma} f\right)^{2}\left(\frac{m_{Q} \sigma-m_{\tilde{Q}} \sigma}{m_{\tilde{Q}} \sigma}\right)^{2} \\
& +\sum_{Q \in \mathcal{D}} \mu(\tilde{Q})\left(a_{Q}-m_{\tilde{Q}}^{\sigma} f\right)^{2} \\
\leq & C W_{1}+W_{2} .
\end{aligned}
$$

We already showed in (21) that $W_{1} \leq C\left\|M_{\sigma} f\right\|_{L^{2}(d \mu)}^{2}$. Recall the fact that given $m$ numbers $a_{j}$, and denoting their mean value by $A=$ $\sum_{j=1}^{m} a_{j} / m$ then, $\sum_{j=1}^{m}\left(a_{j}-A\right)^{2}=C \sum_{j=1}^{m}\left(a_{j}^{2}-A^{2}\right)$. In particular notice that $\sum_{Q \in S(\tilde{Q})} a_{Q} / 2^{n}=m_{\tilde{Q}}^{\sigma} f$. Let $m=2^{n}, A=m_{\tilde{Q}}^{\sigma} f, a_{j}=a_{Q}$; hence

$$
W_{2}=C \sum_{Q \in \mathcal{D}} \mu(\tilde{Q})\left(a_{Q}^{2}-\left(m_{\tilde{Q}}^{\sigma} f\right)^{2}\right) .
$$


Next, add and subtract $m_{Q} \mu\left(m_{Q}^{\sigma} f\right)^{2} / m_{\tilde{Q}} \mu$, then

$$
\begin{aligned}
W_{2}= & \left.C \sum_{Q \in \mathcal{D}} \mu(\tilde{Q})\left(m_{Q}^{\sigma} f\right)^{2}\left(\left(\frac{m_{Q} \sigma}{m_{\tilde{Q}} \sigma}\right)^{2}-\frac{m_{Q} \mu}{m_{\tilde{Q}} \mu}\right)\right) \\
& +C \sum_{Q \in \mathcal{D}}\left(2^{n} \mu(Q)\left(m_{Q}^{\sigma} f\right)^{2}-\mu(\tilde{Q})\left(m_{\tilde{Q}}^{\sigma} f\right)^{2}\right) \\
= & W_{3}+W_{4} .
\end{aligned}
$$

The second summand is a telescoping sum, namely, $W_{4}=\sum_{k}\left(b_{k}-\right.$ $\left.b_{k-1}\right)$, where $b_{k}=\sum_{Q \in \mathcal{D}_{k}\left(Q_{0}\right)} 2^{n} \mu(Q)\left(m_{Q}^{\sigma} f\right)^{2}$, and $\mathcal{D}_{k}\left(Q_{0}\right)$ is the $k^{\text {th }}$ generation of the dyadic decomposition of the base cube $Q_{0}$. Clearly for all $k>0$,

$$
b_{k} \leq C \int_{Q_{0}}\left|M_{\sigma} f(x)\right|^{2} d \mu(x) \leq C\left\|M_{\sigma} f\right\|_{L^{2}(d \mu)}^{2} .
$$

Hence $\left|W_{4}\right| \leq \sup _{k>0}\left|b_{k}\right| \leq C\left\|M_{\sigma} f\right\|_{L^{2}(d \mu)}^{2}$.

Observe that adding and subtracting 1 , we get that,

$$
\begin{aligned}
W_{3}= & C \sum_{Q \in \mathcal{D}} \mu(\tilde{Q})\left(m_{Q}^{\sigma} f\right)^{2}\left(\left(\frac{m_{Q} \sigma}{m_{\tilde{Q}} \sigma}\right)^{2}-1\right) \\
& +C \sum_{Q \in \mathcal{D}} \mu(\tilde{Q})\left(m_{Q}^{\sigma} f\right)^{2} \frac{m_{\tilde{Q}}^{\mu-m_{Q}} \mu}{m_{\tilde{Q}} \mu} \\
= & W_{5}+W_{6} .
\end{aligned}
$$

Notice that

$$
\left(\frac{m_{Q} \sigma}{m_{\tilde{Q}} \sigma}\right)^{2}-1=2 \frac{m_{Q} \sigma-m_{\tilde{Q}} \sigma}{m_{\tilde{Q}} \sigma}+\left(\frac{m_{Q} \sigma-m_{\tilde{Q}} \sigma}{m_{\tilde{Q}} \sigma}\right)^{2} .
$$

Therefore,

$$
\begin{aligned}
W_{5}= & \sum_{Q \in \mathcal{D}} \mu(\tilde{Q})\left(m_{Q}^{\sigma} f\right)^{2}\left(\frac{m_{Q} \sigma-m_{\tilde{Q}} \sigma}{m_{\tilde{Q}} \sigma}\right)^{2} \\
& +2 \sum_{Q \in \mathcal{D}} \mu(\tilde{Q})\left(m_{Q}^{\sigma} f\right)^{2}\left(\frac{m_{Q} \sigma-m_{\tilde{Q}} \sigma}{m_{\tilde{Q}} \sigma}\right) \\
= & W_{1}+2 W_{7} .
\end{aligned}
$$


We already showed that $W_{1} \leq C\left\|M_{\sigma} f\right\|_{L^{2}(d \mu)}^{2}$.

By mean value properties,

$$
\sum_{Q \in S(\tilde{Q})} \frac{m_{Q} \sigma-m_{\tilde{Q}} \sigma}{m_{\tilde{Q}} \sigma}=0
$$

Hence,

$$
W_{7} \leq C \sum_{Q \in \mathcal{D}} \mu(\tilde{Q}) \frac{m_{\tilde{Q}} \sigma-m_{Q} \sigma}{m_{\tilde{Q}} \sigma}\left(\left(m_{Q}^{\sigma} f\right)^{2}-\left(m_{\tilde{Q}}^{\sigma} f\right)^{2}\right) .
$$

Applying the Cauchy-Schwartz inequality, we conclude that

$$
W_{7} \leq C W_{1}^{1 / 2}\left\|S_{\sigma} f\right\|_{L^{2}(d \mu)} \leq C\left\|M_{\sigma} f\right\|_{L^{2}(d \mu)}\left\|S_{\sigma} f\right\|_{L^{2}(d \mu)} .
$$

We are left with the term $W_{6}$. This time

$$
\sum_{Q \in \mathcal{D}} \frac{m_{\tilde{Q}} \mu-m_{Q} \mu}{m_{\tilde{Q}}^{\mu}}=0
$$

Hence,

$$
\begin{aligned}
W_{6} & =C \sum_{\tilde{Q} \in \mathcal{D}} \mu(\tilde{Q}) \sum_{Q \in S(\tilde{Q})} \frac{m_{\tilde{Q}} \mu-m_{Q} \mu}{m_{\tilde{Q}} \mu}\left(\left(m_{Q}^{\sigma} f\right)^{2}-\left(m_{\tilde{Q}}^{\sigma} f\right)^{2}\right) \\
& \leq C\left(\sum_{Q \in \mathcal{D}} \mu(\tilde{Q})\left(\frac{m_{\tilde{Q}} \mu-m_{Q} \mu}{m_{\tilde{Q}} \mu}\right)^{2}\left(m_{Q}^{\sigma} f+m_{\tilde{Q}}^{\sigma} f\right)^{2}\right)^{1 / 2}\left\|S_{\sigma} f\right\|_{L^{2}(d \mu)} \\
& \leq C W_{8}^{1 / 2}\left\|S_{\sigma} f\right\|_{L^{2}(d \mu)} .
\end{aligned}
$$

But $W_{8}$ will be bounded by the $L^{2}(d \mu)$ norm of the maximal function $M_{\sigma}$, if we can show that the sequence $\left(m_{\tilde{Q}} \mu-m_{Q} \mu\right) / m_{\tilde{Q}} \mu$ is a $\mu$ Carleson sequence. If that is the case, we can apply Carleson's lemma as we did for $W_{1}$. But that is exactly the conclusion of Lemma 5 .

Hence $W_{6} \leq C\left\|M_{\sigma} f\right\|_{L^{2}(d \mu)}\left\|S_{\sigma} f\right\|_{L^{2}(d \mu)}$.

Putting together all the estimates we get inequality (22), with constants independent of the base cube $Q_{0}$ and $N$. This finishes the proof of the theorem. 


\subsection{Proof of the weight lemmas.}

Proof of Lemma 4. We are given a $\sigma$-Carleson sequence $\left\{b_{Q}\right\}_{Q \in \mathcal{D}}$; therefore

$$
\sum_{Q \in \mathcal{D}\left(Q_{0}\right)} \sigma(Q) b_{Q}^{2} \leq C \sigma\left(Q_{0}\right), \quad \text { for all } Q_{0} \in \mathcal{D}
$$

This is equivalent to saying that the function $b=\sum_{j} \Delta_{j}^{\sigma} b$ is in dyadic $\operatorname{BMO}(d \sigma)$. Where the weighted difference operator is defined by $\Delta_{j}^{\sigma} b(x)=m_{Q}^{\sigma} b-m_{\tilde{Q}}^{\sigma} b=b_{Q}$, for all $x \in Q \in \mathcal{D}_{j+1}$.

For $\sigma$-doubling and $A_{\infty}^{d}(d \sigma)$ measures it is easy to deduce the John-Niremberg inequality for $\mathrm{BMO}_{d}(d \sigma)$ (see [G, Chapter VI]), and then use it to deduce the equivalent $L^{p}(d \sigma)$ characterizations of dyadic $\operatorname{BMO}(d \sigma)$, namely

$$
\left(\frac{1}{\sigma(Q)} \int_{Q}\left|b-m_{Q}^{\sigma} b\right|^{p} d \sigma\right)^{1 / p} \leq C\|b\|_{\mathrm{BMO}_{d}(d \sigma)}
$$

for all $Q \in \mathcal{D}$. Fix a cube $Q_{0} \in \mathcal{D}$. Define the function

$$
b^{Q_{0}}(x)=\left(b(x)-m_{Q_{0}}^{\sigma} b\right) \chi_{Q_{0}}(x) .
$$

Clearly, $b_{Q}^{Q_{0}}=m_{Q}^{\sigma} b^{Q_{0}}-m_{\tilde{Q}}^{\sigma} b^{Q_{0}}=b_{Q}$ if $Q \in \mathcal{D}\left(Q_{0}\right)$, and zero otherwise.

Then $S_{\sigma} b^{Q_{0}}=\left(\sum_{x \in Q \in \mathcal{D}\left(Q_{0}\right)} b_{Q}^{2}\right)^{1 / 2} ;$ where $S_{\sigma}$ is the weighted square function. Therefore

$$
\int_{Q_{0}} S_{\sigma}^{2} b^{Q_{0}} d \mu=\sum_{Q \in \mathcal{D}\left(Q_{0}\right)} \mu(Q) b_{Q}^{2}
$$

The right hand side is the sum we want to estimate, so it is enough to estimate the integral on the left hand side. It is certainly true by the standard Littlewood-Paley theory that $S_{\sigma}$ is a bounded operator in $L^{p}(d \sigma)$ (not $\left.L^{p}(d \mu) ! !\right)$, for $1<p<\infty$. Hence, noting that $d \mu=\omega_{0} d \sigma$, and using Hölder's inequality we conclude that

$$
\int_{Q_{0}} S_{\sigma}^{2} b^{Q_{0}} d \mu \leq\left\|S_{\sigma}^{2} b^{Q_{0}}\right\|_{L^{p}\left(d \sigma, Q_{0}\right)}\left\|\omega_{0}\right\|_{L^{q}\left(d \sigma, Q_{0}\right)} .
$$


Since by hypothesis $\omega_{0} \in A_{\infty}^{d}(d \sigma)$, then there exists $1<q<\infty$ such that $\omega_{0} \in R H_{q}^{d}(d \sigma)$ (Gëhring's Theorem, see [Ge]), in particular it is true that for $1 / q+1 / p=1$,

$$
\left\|\omega_{0}\right\|_{L^{q}\left(d \sigma, Q_{0}\right)} \leq C \sigma\left(Q_{0}\right)^{-1 / p} \mu\left(Q_{0}\right) .
$$

And also by Littlewood-Paley theory for that particular $p$ we can estimate,

$$
\begin{aligned}
\left\|S_{\sigma}^{2} b^{Q_{0}}\right\|_{L^{p}\left(d \sigma, Q_{0}\right)} & =\left(\int_{Q_{0}} S_{\sigma}^{2 p} b^{Q_{0}} d \sigma\right)^{2 / 2 p} \\
& =\left\|S_{\sigma} b^{Q_{0}}\right\|_{L^{2 p}\left(d \sigma, Q_{0}\right)}^{2} \leq C\left\|b^{Q_{0}}\right\|_{L^{2 p}\left(d \sigma, Q_{0}\right)}^{2} .
\end{aligned}
$$

But by definition,

$$
\begin{aligned}
\left\|b^{Q_{0}}\right\|_{L^{2 p}\left(d \sigma, Q_{0}\right)}^{2} & =C\left(\int_{Q_{0}}\left|b-m_{Q_{0}}^{\sigma} b\right|^{2 p} d \sigma\right)^{1 / p} \\
& \leq C\|b\|_{\mathrm{BMO}(d \sigma)}^{2} \sigma^{1 / p}\left(Q_{0}\right)
\end{aligned}
$$

where the last inequality follows by remark (23).

Therefore, putting all these facts together, we get the desired inequality for all $Q_{0} \in \mathcal{D}$, i.e.

$$
\sum_{Q \in \mathcal{D}\left(Q_{0}\right)} \mu(Q) b_{Q}^{2} \leq C \mu\left(Q_{0}\right)
$$

Therefore the sequence $\left\{b_{Q}\right\}_{Q \in \mathcal{D}}$ is a $\mu$-Carleson sequence, and the lemma is proved.

Proof of Lemma 5. First observe that by hypothesis $\mu$ is a doubling measure, hence $m_{Q} \mu \sim m_{\tilde{Q}} \mu$. Next we want to show that

$$
\sum_{Q \in \mathcal{D}\left(Q_{0}\right)} \mu(Q)\left(\frac{m_{Q} \mu}{m_{\tilde{Q}}^{\mu}}-1\right)^{2} \leq C \mu\left(Q_{0}\right), \quad \text { for all } Q_{0} \in \mathcal{D} \text {. }
$$

Adding and subtracting

$$
\frac{m_{Q} \mu m_{\tilde{Q}} \sigma}{m_{\tilde{Q}} \mu m_{Q} \sigma}
$$


inside the brackets, we can bound the left hand side by

$$
\sum_{Q \in \mathcal{D}\left(Q_{0}\right)} \mu(Q)\left(\frac{m_{Q} \mu m_{\tilde{Q}} \sigma}{m_{\tilde{Q}} \mu m_{Q} \sigma}-1\right)^{2}+\sum_{Q \in \mathcal{D}\left(Q_{0}\right)} \mu(Q)\left(\frac{m_{Q} \mu}{m_{\tilde{Q}} \mu}\right)^{2}\left(\frac{m_{\tilde{Q}} \sigma}{m_{Q} \sigma}-1\right)^{2} .
$$

By hypothesis the sequence

$$
\frac{m_{Q}^{\sigma} \omega_{0}-m_{\tilde{Q}}^{\sigma} \omega_{0}}{m_{\tilde{Q}}^{\sigma} \omega_{0}}=\frac{m_{Q} \mu m_{\tilde{Q}} \sigma}{m_{\tilde{Q}} \mu m_{Q} \sigma}-1
$$

is $\sigma$-Carleson, hence is $\mu$-Carleson by Lemma 4 . Therefore we can control the first summand by a constant multiple of $\mu\left(Q_{0}\right)$.

Similarly the sequence

$$
\frac{m_{Q} \sigma-m_{\tilde{Q}} \sigma}{m_{\tilde{Q}} \sigma} \sim \frac{m_{\tilde{Q}} \sigma}{m_{Q} \sigma}-1
$$

is $d x$-Carleson, hence is $\sigma$-Carleson by Lemma 4 , and is also $\mu$-Carleson, again by Lemma 4 . Hence we can also bound the second summand by a constant multiple of $\mu\left(Q_{0}\right)$, since $\mu$ is doubling. The lemma is proved.

\section{Correspondence $b \leftrightarrow \omega$, and examples.}

In this section we will clarify the correspondence between dyadic doubling $A_{\infty}^{d}$ weights and a subset of $\mathrm{BMO}_{d}$. This has been borrowed from $[\mathrm{KFP}]$. For much more about dyadic weight classes see [B].

Fix a cube $Q_{0}$, without loss of generality we can assume that $Q_{0}=$ $[0,1]^{n}$. With the notation in Section 2.1.2, let

$$
\omega_{N}(x)=\prod_{j=0}^{N}\left(1+\Delta_{j} b(x)\right)
$$

where $b$ is a locally integrable function on $Q_{0}$. Let $\omega=\prod_{j=0}^{\infty}\left(1+\Delta_{j} b\right)$, be the weak limit of the partial products $\left\{\omega_{N}\right\}$. The sequence $\left\{\omega_{N}\right\}$ is a (positive) dyadic martingale when $-1<\Delta_{j} b<1$, since $\int_{Q_{0}}\left(\omega_{k}-\right.$ $\left.\omega_{k-1}\right)(x) d x=0$ and $E\left(\omega: \mathcal{D}_{k}\left(Q_{0}\right)\right)=\omega_{k}$, where $\mathcal{D}_{k}\left(Q_{0}\right)$ is the $\sigma$. field generated by all dyadic cubes contained in $Q_{0}$ of side length $2^{-k}\left|Q_{0}\right|$. 
The necessary and sufficient condition for $\omega$ to be a dyadic doubling measure on $Q_{0}$ is that there exists $0<\varepsilon<1$ such that

$$
\left|\Delta_{j} b(x)\right| \leq 1-\varepsilon, \quad \text { for all } x \in Q_{0}, \text { for all } j \in \mathbb{N} .
$$

(See $[\mathrm{KFP}]$ for a proof.)

We can now give another characterization of dyadic $A_{\infty}^{d}\left(Q_{0}\right)$, assuming doubling.

Theorem 9 (KFP). Let $b$ be a locally integrable function on $Q_{0}$ and $0<\varepsilon<1$, such that $\left|\Delta_{j} b(x)\right| \leq 1-\varepsilon$, for all $x \in Q_{0}$, and for all $j \in \mathbb{N}$. Then the product $\omega=\prod_{j=0}^{\infty}\left(1+\Delta_{j} b\right)$ belongs to doubling $A_{\infty}^{d}\left(Q_{0}\right)$ if and only if there exists constant $C>0$ such that for all $Q^{\prime} \in \mathcal{D}\left(Q_{0}\right)$,

$$
\sum_{Q \in \mathcal{D}\left(Q^{\prime}\right)}|Q|\left(m_{Q} b-m_{\tilde{Q}} b\right)^{2} \leq C\left|Q^{\prime}\right|
$$

For a proof see $[\mathrm{KFP}]$.

We have explained how to compute the weight $\omega$ given the function $b$. It is very easy to check, that given a doubling weight $\omega, m_{Q_{0}} \omega=1$, then,

$$
\Delta_{j} b=\frac{\Delta_{j} \omega}{E_{j} \omega}=\frac{E_{j+1} \omega-E_{j} \omega}{E_{j} \omega} .
$$

After this observation, we see that condition (24) is exactly what we called Buckley's summation condition (18).

Notice also, that the product is nothing more than a telescoping product, since $1+\Delta_{j} b=\dot{E}_{j+1} \omega / E_{j} \omega$; hence $\omega=\prod_{j=0}^{\infty} E_{j+1} \omega / E_{j} \omega$. And equality holds almost everywhere by Lebesgue's theorem, and since $E_{0} \omega=1$.

The martingale condition $E\left(\omega: \mathcal{D}_{k}\left(Q_{0}\right)\right)=\omega_{k}$ is translated into,

$$
E_{k} \omega=\prod_{j=0}^{k-1}\left(1+\Delta_{j} b\right)
$$

Definition. For a locally integrable function $b$ on $Q_{0}$ as in the previous theorem, we will say that $b$ is of $A_{\infty}^{d}$-type on $Q_{0}$ if the doubling weight $\omega=\prod_{j=0}^{\infty}\left(1+\Delta_{j} b\right)$ belongs to $A_{\infty}^{d}\left(Q_{0}\right)$. 
It is known (see [M, vol. 2]), that condition (24) on $b$ means that $b \in \mathrm{BMO}_{d}\left(Q_{0}\right)$. Hence the set of functions $b$ of $A_{\infty}^{d}$-type is a subset of $\mathrm{BMO}_{d}\left(Q_{0}\right)$.

It is known, assuming doubling, that

$$
A_{\infty}^{d}\left(Q_{0}\right)=\bigcup_{p>1} R H_{p}^{d}\left(Q_{0}\right)=\bigcup_{p>1} A_{p}^{d}\left(Q_{0}\right)
$$

Definition. For a locally integrable function $b$ as in the previous theorem, we will say that $b$ is of $R H_{p}^{d}$-type (respectively, $A_{p}^{d}$-type), on $Q_{0}$ if the associated doubling weight is in $R H_{p}^{d}\left(Q_{0}\right)$ (respectively, $A_{p}^{d}\left(Q_{0}\right)$ ).

For the corresponding summation conditions on $b$, see $[\mathrm{B}]$.

Definition. Let $b$ be a locally integrable function on $\mathbb{R}^{n}$, such that $\left|\Delta_{j} b\right| \leq 1-\varepsilon$, for all $j \in \mathbb{Z}$ and some constant $0<\varepsilon<1$. We say that $b$ is of $A_{\infty}^{d}$-type (respectively, $R H_{p}^{d}$ or $A_{p}^{d}$-type) if $b$ is of $A_{\infty}^{d}$-type (respectively, $R H_{p}^{d}$ or $A_{p}^{d}$-type) on $Q$ uniformly for every $Q \in \mathcal{D}$.

Assume from now on that $b$ is a locally integrable function and that there exists $0<\varepsilon<1$ such that for all $j \in \mathbb{Z},\left|\Delta_{j} b\right| \leq 1-\varepsilon$. Let $P_{b}$ be the operator defined formally for $g \in L_{0}^{p}\left(Q_{0}\right)$ by

$$
P_{b} g=\sum_{k=0}^{\infty} \Delta_{k} g \prod_{k+1}^{\infty}\left(1+\Delta_{j} b\right)
$$

If $b$ is of $A_{\infty}^{d}$-type, then the weight $\omega=\prod_{j=0}^{\infty}\left(1+\Delta_{j} b\right)$ is a well defined doubling weight in $A_{\infty}^{d}\left(Q_{0}\right)$; moreover, $\omega / E_{k+1} \omega=\prod_{k+1}^{\infty}\left(1+\Delta_{j} b\right)$, therefore,

$$
P_{b} g=\sum_{k=0}^{\infty} \frac{\omega}{E_{k+1} \omega} \Delta_{k} g=P_{\omega} g,
$$

this last equality by definition of the operator $P_{\omega}$, when restricted to a cube $Q_{0}$. The weight really depends on the base cube $Q_{0}$, but for the estimates we only need uniform bounds on these local versions of $P_{b}$. The bounds are uniform because they only depend on the $R H_{p}^{d}\left(Q_{0}\right)$ constants of the weights, and by definition of $R H_{p}^{d}$-type the local weights have uniform $R H_{p}^{d}\left(Q_{0}\right)$ constants.

We can then study $P_{\omega}$ instead of $P_{b}$, as we claimed. 
Since the paraproduct $\Pi_{b} f$ is bilinear in $b$ and $f$, then, $\lambda \Pi_{b}=\Pi_{\lambda b}$, $\lambda \in \mathbb{N}$. All the algebra (Section 2.2) is valid, and questions about the invertibility of $I-\lambda \Pi_{b}$ are reduced to questions about the weight $\omega_{\lambda}$ corresponding to the function $\lambda b$.

Suppose that $b$ is of $R H_{p}^{d}$-type; we showed that in that case $I-\Pi_{b}$ is invertible in $L^{p}$, with bounded inverse, and that is equivalent to $\omega \in R H_{p}^{d}$ and being doubling. By spectral theory (the resolvent is an open subset of $\mathbb{C}$ ), we know that there exist neighbourhoods of $\lambda=0$ and $\lambda=1$ such that $I-\lambda \Pi_{b}$ is invertible in $L^{p}$. Is this true for every $|\lambda| \leq 1$ ? This question can be translated into a question about weights.

Given $b$ of $R H_{p}^{d}$-type, if $|\lambda| \leq 1$ then certainly $\lambda b$ is of $A_{\infty}^{d}$-type and $\left|\Delta_{j}(\lambda b)\right| \leq 1-\varepsilon$. We are saying that under this correspondence, multiplication by $|\lambda| \leq 1$ preserves doubling and $A_{\infty}^{d}$ weights. This is different from the standard correspondence $\tilde{\omega}=e^{b}$ (for example, consider $b=\log |x|$, and $\lambda=-1$, then $\tilde{\omega}_{\lambda}=1 /|x|$, which is not even locally integrable, not to say in $A_{\infty}^{d}$.) weights.

The question is if multiplication by $|\lambda| \leq 1$ preserves doubling $R H_{p}^{d}$

For $-1<\lambda<0$ the answer is negative, and it is the content of Theorem III. We would like to know what happens for $0<\lambda<1$.

Proof of Theorem III. We will produce examples stemming from the prototype function in BMO, namely, $C \log (1 /|x|)$. It is enough to consider a dyadic version of this functions, in dimension $n=1$. After being requested by electronic mail, S. Buckley produced the same sort of examples.

Fix $I_{0}=[0,1]$. Let $I_{k}=\left(2^{-k}, 2^{-k+1}\right], k \geq 1$. For $0<\varepsilon<2$, define the step function $b_{\varepsilon}(x)=k(1-\varepsilon), x \in I_{k}$. Certainly $\left|\Delta_{j} b_{\varepsilon}\right| \leq|1-\varepsilon|$, and $b_{\varepsilon} \in \mathrm{BMO}_{d}\left(I_{0}\right)$. Hence $\omega_{\varepsilon}=\prod_{j=0}^{\infty}\left(1+\Delta_{j} b_{\varepsilon}\right)$ is a doubling $A_{\infty}^{d}$ weight.

REMARK. This is not the case for the standard correspondence, $\tilde{\omega}=e^{b}$; for example, let $\varepsilon=1-\log 2$, then $\tilde{\omega}_{\varepsilon}$ will correspond to the dyadic version of $1 /|x|$.

We can say more about $b_{\varepsilon}$; it corresponds to the dyadic version of the function $\alpha(\varepsilon) \log (1 /|x|)$, where $\alpha(\varepsilon)=(1-\varepsilon) / \log 2$. (Hence the remark, for that $\varepsilon, \alpha(\varepsilon)=1$.) The weight $\omega_{\varepsilon}$ corresponding to $b_{\varepsilon}$ can be explicitely computed, $\omega_{\varepsilon}=C(2-\varepsilon)^{k}, x \in I_{k}$. Hence, $\omega_{\varepsilon}$ corresponds to the continuous weight $|x|^{\gamma(\varepsilon)}$, where $\gamma(\varepsilon)=-\log (2-\varepsilon) / \log 2$. Observe 
that $-1<\gamma(\varepsilon)<\infty$ for $0<\varepsilon<2$. We captured the full range of prototype $A_{\infty}^{d}$ weights,.namely $|x|^{\gamma}, \gamma>-1$. It is easy to check that a power $|x|^{\gamma}$ belongs to $R H_{p}^{d}$ if and only if $\gamma>-1 / p, 1<p<\infty$. Hence, solving for $0<\varepsilon<2$ the inequality $\gamma(\varepsilon)>-1 / p$, we get that only for $2-2^{1 / p}<\varepsilon<2, \omega_{\varepsilon} \in R H_{p}^{d}$. Given $-1<\lambda<0$, there exists $1<p<\infty$ such that $\lambda<1-2^{1 / p}$. Fix such $p$. Observe that $\lambda b_{\varepsilon}=b_{\varepsilon(\lambda)}$, where $\varepsilon(\lambda)=1-(1-\varepsilon) \lambda$. If we could find $2-2^{1 / p}<\varepsilon<2$ such that $\varepsilon(\lambda) \leq 2-2^{1 / p}$ then we would be done, because $\omega_{\varepsilon} \in R H_{p}^{d}$ but $\omega_{\varepsilon(\lambda)}$ is not in $R H_{p}^{d}$. We can certainly find such $\varepsilon$; any $2^{1 / p}<\varepsilon<2$ will do the job. Hence we find function $b=b_{\varepsilon}$ of $R H_{p}^{d}$-type so that $\lambda b=b_{\varepsilon(\lambda)}$ is not. In particular, by Theorems 1 and 2 , we conclude that $I-\lambda \Pi_{b}$ does not have a bounded inverse in $L^{p}$, hence $\lambda \Pi_{b}$ cannot be a contraction in $L^{p}$, i.e. $\left\|\Pi_{b}\right\|_{p, p} \geq 1 /|\lambda|$.

This finishes the proof of Theorem III.

REMARK. For these examples, multiplication by $0<\lambda<1$ preserves the $R H_{p}^{d}$-type condition on $I_{0}$.

\section{References.}

[B] Buckley, S., Summation conditions on weights. Michigan Math. J. 40 (1993), 153-170.

[CF] Coifman, R. R. and Fefferman, C., Weighted norm inequalities for maximal functions and singular integrals. Studia Math. 51 (1974), 241-250.

[Ch] Christ, M., Lectures on singular integral operators. Regional conferences series in Math. Amer. Math. Soc. 77 (1990).

[D] David, G., Wavelets and Singular Integrals on Curves and Surfaces. Lecture Notes in Math. 1465, Springer-Verlag, 1991.

[G] Garnett, J., Bounded Analytic Functions. Academic Press, 1981.

[Ga] Garsia, A. M., Martingale inequalities, Seminar Notes on Recent Progress. Benjamin, 1973.

[GC-RF] García-Cuerva, J. and Rubio de Francia, J. L., Weighted norm inequalities and related topics. North Holland, 1985.

[Ge] Gehring, F. W., The $L^{p}$ integrability of the partial derivatives of a quasiconformal mapping. Acta Math. 130 (1973), 265-277.

[KFP] Kenig, C., Fefferman, R. and Pipher, J., The theory of weights and the Dirichlet problem for elliptic equations. Annals of Math. 134 (1991), 65-124.

[M] Meyer, Y., Ondelettes et Opérateurs, Vol. I, II and III. Herman, 1990. 
[P] Pereyra, M. C., Sobolev spaces on Lipschitz curves, paraproducts, and related operators. Ph.D. Thesis, Yale University, 1993.

[P1] Pereyra, M. C., Sobolev spaces on Lipschitz curves. To appear in Pacific J. Math.

[R] Rochberg, R., Size estimates for eigenvectors of singular integral operators. To appear in Amer. J. Math.

[S] Stein, E., Topics in harmonic analysis, related to the Littlewood-Paley theory. Annals of Mathematical Studies 63, Princeton Univ. Press, 1970.

Recibido: 30 de junio de 1.993

María Cristina Pereyra Department of Mathematics Yale University New Haven, CT 06520, U.S.A.

and

Department of Mathematics Princeton University Princeton, NJ 08544, U.S.A. 